SLAC-PUB-245

November 1966

\title{
ANALYTIC CONTINUATION OF GROUP REPRESENTATIONS - $\mathrm{V}^{*}$ \\ Robert Hermann \\ Stanford Linear Accelerator Center, Stanford, California
}

\begin{abstract}
The connection between analytic continuation of group representations and analytic continuation of their matrix elements is discussed, together with some related problems concerning the group-theoretic nature of the S-matrix, and the asymptotic behavior of the special functions of mathematical physics.
\end{abstract}

(To be submitted to Comm. of Math. Phys.)

\footnotetext{
Work supported by U.S. Atomic Energy Commission
} 


\section{ANALYTIC CONTINUATION OF GROUP REPRESENTATIONS - V \\ Robert Hermann \\ Stanford Linear Accelerator Center, Stanford, California}

\section{INTRODUCTION}

So far in this series we have dealt with analytic continuation at the "infinitesimal" level, i.e., we have considered "analytic continuation" of Lie algebra representations, trusting to the known relations between Lie algebra and Lie group representations to provide us (at least implicitly) with corresponding facts about continuation of group representations. Needless to say, it is at the group level that the most interesting application to physics are to be found: For example, the "special functions" of mathematical physics occur as matrix elements of various group representations. The general problem of reducing the tensor products of representations into irreducible components and generalized "WignerEckart" theorems is of prime importance for the application of group theory to elementary particle physics.

The interesting problems concerning analytic continuation of group representations and their matrix elements lead to additional degrees of complexity beyond those we have encountered, since questions of analysis and geometry as well as algebra appear. For example, one of our central problems can be described as follows: Suppose for each value of the parameter $\lambda, t \rightarrow U_{\lambda}(t)$ is a one-parameter group of unitary transformations of a Hilbert space H. Consider two elements $\psi, \psi^{\prime}$ of $\mathrm{H}$, and the matrix element.

$$
\begin{gathered}
f(t, \lambda)=\left\langle\psi\left|U_{\lambda}(t)\right| \psi^{\prime}\right\rangle \\
-1-
\end{gathered}
$$


One can ask various questions about the analyticity of this function of $t$ and $\lambda$, and its asymptotic behavior as $t$ and/or $\lambda$ go to infinity. For example, as we will show, the asymptotic formula:

$$
\mathrm{P}_{\ell}(\mathrm{z}) \sim \text { constant } \mathrm{xz} \mathrm{z}^{\ell} \text { as } \mathrm{z} \rightarrow \infty
$$

that plays such an important role in high-energy behavior of the scattering amplitude is a typical case. (See [11] , Chapter 14 for work on this problem from a slightly different point of view from that presented here.)

The ideas of Dashen and Gell-Mann [2] concerning the "infinite momentum" limit also are of this nature, although we shall not be explicitly considering this case in this paper. The formula

$$
\lim _{\ell \rightarrow \infty} \overline{\mathrm{P}}_{\ell}^{j}\left(\cos \frac{t}{\ell}\right)=J_{j}(t)
$$

of Inonu and Wigner [13] is also in this pattern.

In addition to these concrete problems there is also much work to be done in developing the relation between analytic continuation of group representations and group cohomology, a subject pioneered by Nijenhuis and Richardson [16]. (Note that the relation between group cohomology and Lie algebra cohomology has been developed by W. van Est [4]). Again, there is an interesting and useful interrelation between various abstract questions and concrete problems that appear in physics. In fact, there is a situation here that is almost unparalleled since the nineteenth century: interesting physical questions suggest interesting mathematical ones, and conversely. We shall present topics in this paper that proceed in the direction of elucidating these connections. 


\section{GENERAL FACTS ABOUT MATRIX ELEMENTS}

Let $\mathrm{G}$ be a group, $\mathrm{H}$ a Hilbert space, and $\rho$ a representation of $\mathrm{G}$ by operators on $\mathrm{H}$. Denote elements of $\mathrm{H}$ by $\psi, \psi^{\prime}$, etc., the Hermitian inner product by $\left\langle\psi \mid \psi^{\prime}\right\rangle$. (We use the physicist's rule:

$$
\begin{aligned}
& <\psi\left|\lambda \psi^{\prime}\right\rangle=\lambda\left\langle\psi \mid \psi^{\prime}\right\rangle \\
& <\lambda \psi\left|\psi^{\prime}\right\rangle=\lambda^{*}\left\langle\psi \mid \psi^{\prime}\right\rangle
\end{aligned}
$$

for each complex number $\lambda . \quad \lambda^{*}$ denotes the complex conjugate.)

For fixed elements $\psi, \psi^{\prime}$ of $\mathrm{H}$, the function $\mathrm{g} \rightarrow\left\langle\psi^{\prime}\right| \rho(\mathrm{g}) \psi>=\mathrm{f}(\mathrm{g})$ is the matrix element corresponding to $\psi$ and $\psi^{\prime}$. In [11], Chapter 9, one will find a short discussion from a slightly different point of view of the relation between the matrix elements as functions on $G$ and the representations themselves. (This, in essence, is the theory of "induced representations.") Let $\rho(g)$ " be the adjoint operator to $\rho(g)$

$$
\left\langle\psi^{\prime}\right| \rho(g) \psi>=\left\langle\rho(g) * \psi^{\prime} \mid \psi\right\rangle
$$

Since $(A B))^{*}=B^{*} A^{*}$ for operators $A, B$, we must define the "dual" representation $\rho^{*}$ by $\mathrm{G}$ by operators on $\mathrm{H}$ via the formula:

$$
\rho^{*}(g)=\rho\left(g^{-1}\right)^{*}
$$

Then

$$
\begin{aligned}
\mathrm{f}(\mathrm{g}) & =\left\langle\psi^{\prime} \mid \rho(\mathrm{g}) \psi\right\rangle=\left\langle\rho(\mathrm{g})^{*} \psi^{\prime} \mid \psi\right\rangle \\
& =\left\langle\psi \mid \rho(\mathrm{g})^{*} \psi^{\prime}\right\rangle^{*} \\
& =\left\langle\psi \mid \rho^{*}\left(\mathrm{~g}^{-1}\right) \psi^{\prime}\right\rangle^{*}
\end{aligned}
$$


This formula provides the connection between the matrix elements of $\rho$ and those of $\rho^{*}$.

Let $K$ be a subgroup of $G$. Suppose $\psi_{1}, \ldots, \psi_{n}$ are elements of $H$ that transform among themselves by $\rho(K)$, i. e. ,

$$
\rho(k) \dot{\psi_{i}}=\sigma_{\mathrm{ji}}(\mathrm{k}) \psi_{\mathrm{j}} \text { for } \mathrm{k} \in \mathrm{K}
$$

$(1 \leq i, j, \ldots . n$; summation convention in force.)

$\mathrm{k} \rightarrow\left(\sigma_{i j}(\mathrm{k})\right)$ is an $\mathrm{n} \times \mathrm{n}$ matrix representation of $\mathrm{K}$ that we will denote by $\sigma$. Let $\sigma^{\mathrm{T}}$ be the transposed representation, i. e.,

$$
\left(\sigma^{\mathrm{T}}(\mathrm{k})\right)_{\mathrm{ji}}=\sigma\left(\mathrm{k}^{-1}\right)_{\mathrm{ij}}
$$

Let $\mathrm{f}_{m}(\mathrm{~g})=\left(\mathrm{f}_{1}(\mathrm{~g}), \ldots, \mathrm{f}_{\mathrm{n}}(\mathrm{g})\right)$, with $\mathrm{f}_{\mathrm{i}}(\mathrm{g})=\left\langle\psi^{\prime} \mid \rho(\mathrm{g}) \psi_{\mathrm{i}}\right\rangle \ldots$

Regard $f_{m}$ as a map. $\mathrm{G} \rightarrow \mathrm{C}^{\mathrm{n}}$.

For $k \in K$

$$
\begin{aligned}
\mathrm{f}_{\mathrm{i}}(\mathrm{gk}) & =\left\langle\psi^{\prime} \mid \rho(\mathrm{g}) \rho(\mathrm{k}) \psi_{\mathrm{i}}\right\rangle \\
& =\sigma_{\mathrm{ji}}(\mathrm{k})<\psi^{\prime} \mid \rho(\mathrm{g}) \psi_{\mathrm{j}}> \\
& =\sigma^{\mathrm{T}}\left(\mathrm{k}^{-1}\right)_{\mathrm{ij}} \mathrm{f}_{\mathrm{j}}(\mathrm{g})
\end{aligned}
$$

Hence,

$$
f(g k)=\sigma^{T}\left(k^{-1}\right) \underline{f}(g)
$$

i. e., $f$ is a cross section of the vector bundle on the homogeneous space $G / K$ determined by the linear representation $k \rightarrow \sigma(k)$ of $K$. (See [11] for background on vector bundles.) We shall denote the space of cross sections of this vector bundle, i.e., the space of mappings $G \rightarrow C^{n}$ satisfying $(2.1)$, by $\Gamma\left(\sigma^{\mathrm{T}}\right)$. Note in particular, that if $\mathrm{n}=1$ and $\sigma_{11}(\mathrm{k})=1$, then

$$
\underline{f}(\mathrm{gk})=\underline{\mathrm{f}}(\mathrm{g})
$$

i.e., $f$ determines a function on $\mathrm{G} / \mathrm{K}$. 
Now, hold $\psi_{1}, \ldots, \psi_{\mathrm{n}}$ fixed, and consider $\psi^{\mathrm{h} \rightarrow \mathrm{f}}$ as a mapping $H \rightarrow \Gamma\left(\sigma^{\mathrm{T}}\right)$. Note that it is antilinear, i.e., sums go into sums, but

$$
\lambda \psi^{\prime} \text { goes into } \lambda^{*} \underline{\underline{\prime}}
$$

There is a representation of $\mathrm{G}$ by lincar transormations on $\Gamma\left(\sigma^{\mathrm{T}}\right)$ : The transform of $f: G \rightarrow C^{n}$ by an element $g_{0} \in G$ is the function

$$
g \rightarrow f\left(g_{0}^{-1} g\right)
$$

If $\mathrm{f}_{\mathbf{j}}(\mathrm{g})=\left\langle\psi^{\prime}\right| \rho(\mathrm{g}) \psi_{\mathbf{j}}>$, then

$$
\begin{aligned}
\mathrm{f}_{\mathrm{j}}\left(\mathrm{g}_{\mathrm{o}}^{-1} \mathrm{~g}\right) & =\left\langle\psi^{\prime}\right| \rho\left(\mathrm{g}_{0}^{-1}\right) \rho(\mathrm{g}) \psi_{\mathrm{j}}> \\
& =<\rho\left(\mathrm{g}_{\mathrm{o}}^{-1}\right)^{*} \psi^{\prime}\left|\rho(\mathrm{g}) \psi_{\mathrm{j}}\right\rangle \\
& =\left\langle\rho^{*}\left(\mathrm{~g}_{\mathrm{o}}\right) \psi^{\prime} \mid \rho(\mathrm{g}) \psi_{\mathrm{j}}\right\rangle,
\end{aligned}
$$

i. e., the vector $\rho^{*}\left(\mathrm{~g}_{\mathrm{O}}\right) \psi^{*}$ goes over into the transform of $\mathrm{f}$ by $\mathrm{g}_{0}$. Summing up, we have proved:

Theorem 2.1 Suppose $\psi_{1}, \ldots, \psi_{\mathrm{n}}$ are elements of $\mathrm{H}$ transforming under the subgroup $\mathrm{K}$ by an $\mathrm{n} \times \mathrm{n}$ matrix representation $\sigma$. Then the correspondence

$$
\psi^{\prime} \rightarrow \mathrm{f}: \mathrm{g} \rightarrow\left(\left\langle\psi^{\prime} \mid \rho(\mathrm{g}) \psi_{\mathrm{i}}\right\rangle\right)
$$

defines an antilinear map $\mathrm{H} \rightarrow \Gamma\left(\sigma^{\mathrm{T}}\right)$ that is an intertwining map with respect to the given linear action of $G$ on $H$ and the left translation action of $G$ on $\Gamma\left(\sigma^{*}\right)$

Theorem 2.1 sums up many of the special properties of "matrix elements" of representations that are uscful in physics. Often it is further possible to express the matrix-element functions $f: G \rightarrow C^{n}$ as products corresponding to 
certain product-subgroup decompositions of G. As an example, let us consider the case where $\mathrm{K}$ is a compact, symmetric subgroup of $\mathrm{G}$, with $\mathrm{G}$ semisimple and connected, i.e., $\mathrm{G}=\mathrm{K} \odot \underset{\mathrm{m}}{\mathrm{P}}$, with

$$
[\underline{\mathrm{K}}, \mathrm{P}] \subset \underset{m}{\mathrm{P}},[\underline{\mathrm{P}}, \mathrm{P}] \subset \mathrm{K}
$$

Let $\mathrm{A}$ be a maximal abelian subalgebra of $\mathrm{P}$, and let $\mathrm{A}$ be the connected abelian subgroup of $\mathrm{G}$ generated by $\mathrm{A}$. Then, it is a general theorem [9] that:

$$
\mathrm{G}=\mathrm{KAK}
$$

(This means that every element $g \in G$ can be written (not necessarily uniquely) as a product $k_{1} a k_{2}$, with $k_{1}, k_{2} \in K$, a $\left.\in A\right)$. In case $G=S O(3, R)$, this is just the Euler angle description of rotations, so it may be regarded as a generalization of the Euler angle construction for an arbitrary semisimple Lie group.

Then,

$$
\begin{aligned}
\mathrm{f}_{\mathrm{i}}\left(\mathrm{k}_{1} a \mathrm{k}_{2}\right) & =\left\langle\psi^{\prime}\right| \rho\left(\mathrm{k}_{1}\right) \rho(a) \rho\left(\mathrm{k}_{2}\right) \psi_{\mathrm{i}}> \\
& =\left\langle\rho\left(\mathrm{k}_{1}\right)^{*} \psi^{\prime}\right| \rho\left(\mathrm{a}_{1}\right) \rho\left(\mathrm{k}_{2}\right) \psi_{\mathrm{i}}> \\
& =\sigma\left(\mathrm{k}_{2}\right)_{\mathrm{ji}}\left\langle\rho\left(\mathrm{k}_{1}\right)^{*} \psi^{\prime} \mid \rho\left(\mathrm{a}_{1}\right) \psi_{\mathrm{j}}\right\rangle .
\end{aligned}
$$

Now, we can decompose the action of $\rho^{*}(\mathrm{~K})$ on II. For examplc, suppose $\left(\psi_{\mu}^{\prime}\right)$ are elements transforming as follows:

$$
\rho^{*}(\mathrm{k})\left(\psi_{\mu}^{\prime}\right)=\gamma_{\nu \mu}(\mathrm{k}) \psi_{\nu}^{\prime}
$$

$(1 \leq \mu, \nu, \ldots \leq \mathrm{m} ;$ summation convention on these indices). Put:

$$
\mathrm{f}_{\mathrm{i}}^{\mu}=\left\langle\psi_{\mu}^{\prime} \mid \rho(\mathrm{g}) \psi_{\mathrm{i}}\right\rangle
$$

Then,

$$
\mathfrak{f}_{i}^{\mu}(\mathrm{g})=\sigma\left(\mathrm{k}_{2}\right)_{\mathrm{ji}} \gamma_{\nu \mu}\left(\mathrm{k}_{\mathrm{i}}^{-1}\right) \mathrm{f}_{\mathrm{j}}^{\nu}(\mathrm{a})
$$


Thus, we see that the matrix elements are determined by:

(a) The decomposition of $\rho(\mathrm{K})$ and $\rho^{*}(\mathrm{~K})$ into irreducible representations.

(b) The matrix elements restricted to the subgroup A, which may be expected to satisfy certain differential equations.

Since this sort of straightforward description of the matrix elements should be familiar to the reader who has studied special representations of special groups, i.e., $\mathrm{G}=\mathrm{SO}(3, \mathrm{R})$ or $\mathrm{G}=\mathrm{SO}(2,1)$ or $\mathrm{SL}(2, \mathrm{C})$, we will leave it at this point.

\section{INTEGRAL REPRESENTATIONS FOR MATRIX ELEMENTS}

We will continue to review certain general facts about matrix elements that are merely general versions of calculations well known in special cases. Let us continue with a representation $\rho$ of a group $G$ on a Hilbert space $H$. In the last section, we supposed that $\mathrm{H}$ was given abstractly. Let us now suppose that it is determined as a space of cross sections of a vector bundle on which $\mathrm{G}$ acts. Consider a vector bundle

$$
\pi: \mathrm{E} \rightarrow \mathrm{MI} \text {. }
$$

$\left(M\right.$ is a manifold, $\pi(E)=M$. For each $p \in M$, the fibre $\pi^{-1}(p)$ is a complex vector space). Suppose G acts linearly on E. (This means: G acts as a transformation group on $\mathrm{E}$ and $\mathrm{M}$; with $\mathrm{g} \pi(\nu)=\pi \mathrm{g}(\nu)$ for $\mathrm{g} \in \mathrm{G}, \nu \in \mathrm{E}$. Transformation by $g$ maps the vector space $\pi^{-1}(p)$ linearly onto $\pi^{-1}(g p)$, for each $p \in$ M.) Let $\Gamma(E)$ be the space of cross sections. The action of $G$ on $M$, together with a multiplier system $\mathrm{m}(\mathrm{g}, \mathrm{p})$ determines a representation $\rho$ of $\mathrm{G}$ in $\Gamma(\mathrm{E})$.

For $\psi \in \Gamma(E), g \in G, p \in M$,

$$
\rho(\mathrm{g})(\psi)(\mathrm{p})=\mathrm{m}(\mathrm{g}, \mathrm{p}) \mathrm{g} \psi\left(\mathrm{g}^{-1} \mathrm{p}\right)
$$


For $g \in G, p \in M, m(g, p)$ is a linear map of the fibre $\pi^{-1}(p)$ into itself. It satisfies the functional equation

$$
\begin{aligned}
& \mathrm{m}\left(\mathrm{g}_{0} \mathrm{~g}_{1}, \mathrm{p}\right)=\mathrm{m}\left(\mathrm{g}_{\mathrm{o}}, \mathrm{p}\right) \mathrm{g}_{\mathrm{o}} \mathrm{m}\left(\mathrm{g}_{1}, \mathrm{~g}_{\mathrm{o}}^{-1} \mathrm{p}\right) \mathrm{g}_{\mathrm{o}}^{-1} \\
& \text { for } \mathrm{g}_{0}, \mathrm{~g} \in \mathrm{G}, \tau \in \mathrm{M} .
\end{aligned}
$$

(In fact this is just the relation necessary to have $\rho\left(\mathrm{g}_{0} \mathrm{~g}_{1}\right)=\rho\left(\mathrm{g}_{0} \mid \rho\left(\mathrm{g}_{1}\right)\right)$, i. e. , $\rho$ is a representation.)

Let us make $\Gamma(\mathrm{E})$ into a Hilbert space in the following way: Suppose given, for each $p \in M$, a Hermitian inner product (,) on the fibre $\pi^{-1}(p)$. For $\psi, \psi^{\prime} \in \Gamma(\mathrm{E}), \mathrm{p} \rightarrow\left(\psi(\mathrm{p}), \psi^{\prime}(\mathrm{p})\right)$ is then a function on $\mathrm{M}$. Let $\mathrm{dp}$ be a volume element for the manifold M. Define:

$$
\left\langle\psi \mid \psi^{r}\right\rangle=\int_{M}\left(\psi(p), \psi^{\prime}(p)\right) d p
$$

The conditions that the representation $\rho$ be unitary are worked out in [10], Chapter 9.

It is now obviously a routine matter to work out an integral representation for the matrix elements:

$$
\begin{aligned}
\left\langle\rho(\mathrm{g})(\psi) \mid \psi^{r}\right\rangle & =\int_{\mathrm{M}}\left(\rho(\mathrm{g})(\psi)(\mathrm{p}), \psi^{\prime}(\mathrm{p})\right) \mathrm{dp} \\
& =\int_{\mathrm{M}}\left(\mathrm{m}(\mathrm{g}, \mathrm{p}) \mathrm{g} \psi\left(\mathrm{g}^{-1} \mathrm{p}\right), \psi^{\prime}(\mathrm{p})\right) \mathrm{dp}
\end{aligned}
$$

As a simple illustration, suppose $G$ is semisimple, non-compact, $M=G / H$, where $H$ is one of the "boundary homogeneous spaces" of Furstenberg [ $0,8,11]$, $\Gamma(E)$ is the space of complex-valued functions on $M$, i. e., the vector bundle $E$ 
is just the product $M \times C$. Choose $m(g, p)$ as follows:

$$
\mathrm{m}(\mathrm{g}, \mathrm{p})=\mathrm{J}_{\mathrm{g}^{-1}}(\mathrm{p})^{\lambda}
$$

where $J_{\mathbf{g}}(p)$ is the Jacobian of the transformation $p_{-}, g p$. with respect to the volume element $\mathrm{dp}, \lambda$ is a fixed complex number. Then

$$
\left\langle\rho(\mathrm{g}) \psi \mid \psi^{\prime}\right\rangle=\int_{\mathrm{M}} \mathrm{J}_{\mathrm{g}^{-1}}^{-\lambda}(\mathrm{p}) \psi\left(\mathrm{g}^{-1} \mathrm{p}\right)^{*} \psi^{\prime}(\mathrm{p}) \mathrm{dp}
$$

Now, one basic property of these boundary homogeneous spaces is that $K$, the maximal compact subgroup of $G$, acts transitively on them. Thus, we can suppose dp chosen as the unique (up to a scaler multiple) volume element which is invariant under $\mathrm{K}$, i. e. ,

$$
J_{k}(p)=1 \text { for } k \in K, \tau \in M
$$

In particular, if $\psi(p) \equiv 1$, then

$$
\rho(k)(\psi)=\psi \text { for all } \mathrm{k} \in \mathrm{K}
$$

and the matrix element

$$
\mathrm{g} \rightarrow<\rho(\mathrm{g})(\psi) \mid \psi^{\prime}>=\mathrm{f}(\mathrm{g})
$$

is a function on the symmetric space $G / K$, i.e.,

$$
<\rho(\mathrm{gk})(\psi)\left|\psi^{\prime}\right\rangle=\left\langle\rho(\mathrm{g}) \psi \mid \psi^{\prime}\right\rangle \quad \text { for } \mathrm{k} \in \mathrm{K}, \mathrm{g} \in \mathrm{G} \text {. }
$$

Then, we have:

$$
f(g)=\int_{M} J_{g^{-1}}^{-\lambda}(p) \psi^{\prime}(p) d p
$$

This explains why the spaces of the type $M$ are called "boundary homogeneous spaces" for the symmetric space $G / K$. An important class of functions 
on $G / K$, namely the matrix elements of the "class 1 " representations of $G$, i. e. , these in which $\mathrm{K}$ has an invariant vector $[9]$, are exhibited in a natural way as integral transforms of. functions on M. (Of course, there is also a geometric motivation: They appear as parts of the boundary when the spaces $\mathrm{G} / \mathrm{K}$ are compactified $[4,10]$ ). These remarks take on a special importance for physics when one understands that most of the special functions of mathematical physics appear as matrix elements with respect to representations of a relatively small number of groups.

It is important to notice also that these considerations are not restricted to unitary representations of $G$. Thus, we may start off with a representation $\rho_{\lambda}$ of a real Lie group $\mathrm{G}$ on a Hilbert space $\mathrm{H}$, with the representation depending on a parameter set $\lambda$. The matrix elements are then functions on $G$, depending also on the parameter $\lambda$. Let $G_{c}$ be the complexification of $G$, i. e., the real parameters of the group are considered as complex, and $G$ is a subgroup of $G_{c}$. (Technically, one proceeds as follows: Let $G$ be the Lie algcbra of G. Let $\mathrm{G}_{\mathrm{m}}=\mathrm{G}+\mathrm{i} \mathrm{G}$ be its complexification as a Lie algebra. Consider $\mathrm{G}_{\mathrm{m}}$ also as a real Lic algebra of twice the dimension of $G$, and with $G$ as a subalgebra. A complexification of $G$ is a Lic group $G_{c}$ whose Lie algcbra is $G_{m} c$, containing $G$ as a subgroup in such a way as to give use to the given imbedding of $G$ in $G$ An arbitrary Lie group may not have a complexification in this technical sense although of course all the "classical" groups do, i. c., $\mathrm{SO}(\mathrm{n}, \mathrm{R}) \rightarrow \mathrm{SO}(\mathrm{n}, \mathrm{C})$, $\mathrm{SU}(\mathrm{n}) \rightarrow \mathrm{SL}(\mathrm{n}, \mathrm{C}), \mathrm{SO}(\mathrm{p}, \mathrm{q}) \rightarrow \mathrm{SO}(\mathrm{p}+\mathrm{q}, \mathrm{C})$, etc. Let us suppose that the representation $\rho$ may be extended to a representation $\rho$ of $G_{C}$ on the same Hilbert space. Then, the matrix elements $g \rightarrow\left\langle\psi^{\prime} \mid \rho(g) \psi\right\rangle$ will be complex-analytic with respect to the complex analytic parameters for $G_{c}$ if the following condition is satisfied.

$$
\rho(\mathrm{iX})=\mathrm{i} \rho(\mathrm{X}) \text { for } \mathrm{X} \in \mathrm{G}_{\mathrm{C}}
$$


Of course, this condition implies that the representation cannot be extended to be unitary on $G_{c}$. Conversely, 3.5 can be used to extend $\rho$ from $G_{m}$ to $G_{m}$. Even though $\rho$ may be "integrated" to give a global representation of $G$, the extended representation of $\mathrm{G}_{\mathrm{c}}$ may not necessarily give such a global representation of $G_{c}$ : This is a group-theoretical reflection of the fact that the matrix elements may have singularities when an attempt is made to analytically continue them to all of $G_{c}$. At any rate, Nelson's theory of "analytic vectors" [15] does guarantee that $\rho$ can be extended to a neighborhood of the identity in $\mathrm{G}_{\mathrm{c}}$, which means that the matrix element

$$
<\psi^{\prime} \mid \rho(\mathrm{g}) \psi>
$$

may be defined when $g$ is sufficiently near the identity in $G_{c}$. Unfortunately, there seem to be very few examples of this phenomenon worked out explicitly in the literature to serve as a guide to a thorough analysis. We will work out the case $G=S T,(2, R)$ in Section 10 .

\section{LEGENDRE FUNCTIONS AND THE GELL-MANN FORMULA}

It is well-known that the Legendre functions appear as matrix elements of representations of $\mathrm{SO}(3, \mathrm{R})$ and its noncompact real form $\mathrm{SO}(2,1)$. The "GellMann formula" works particularly well for these groups; we will now show how it gives information about their matrix elements:

Let $G$ be the Lie algebra of $S O(3, R)$. It is generated by elements $X, Y, Z$, - satisfying

$$
[\mathrm{Z}, \mathrm{X}]=\mathrm{Y} ;[\mathrm{Z}, \mathrm{Y}]=\mathrm{X} \quad[\mathrm{X}, \mathrm{Y}]=\mathrm{Z}
$$


(For example, in the physicist's notation,

$$
Z=J_{z}, X=J_{x}, Y=J_{y} \text { where } \vec{J}=\left(J_{x}, J_{y}, J_{z}\right) \text { is the angular }
$$

momentum vector.) The Casimir operator is

$$
\Delta=\mathrm{X}^{2}+\mathrm{Y}^{2}+\mathrm{Z}^{2}
$$

Consider a finile dimensional representation $\rho_{\ell}$ where $\Delta$ has the value $-\ell(\ell+1)$. Then, $\rho(Z)$ has the eigenvalues $-i \ell, \ldots$, i $\ell$, with corresponding eigenvectors $\psi_{-\lambda}, \ldots, \psi_{\lambda}$.

The functions

$$
\mathrm{f}_{\ell}^{\mathrm{j}}(\mathrm{t})=\left\langle\psi_{\mathrm{O}} \mid \rho_{\ell}(\operatorname{Exp} \mathrm{tX})\left(\psi_{\mathrm{j}}\right)\right\rangle-\ell \leq \mathrm{j} \leq \ell
$$

are essentially the Legendre polynomials. (At least after taking account of normalization, and putting $\mathrm{z}=\cos \mathrm{t}$.)

In [12 part I], we have indicated a way of exhibiting these representations by starting with a representation of one of its contractions, namely the group of rigid motions in the plane. Its Lie algebra is generated by elements $Z, X^{\prime}, Y^{\prime}$, with structure relations:

$$
\left[\mathrm{Z}, \mathrm{X}^{\prime}\right]=\mathrm{Y}^{\prime} ;\left[\mathrm{Z}, \mathrm{Y}^{\prime}\right]=-\mathrm{X}^{\prime}\left[\mathrm{X}^{\prime}, \mathrm{Y}^{\prime}\right]=0
$$

Let $\phi(\mathrm{Z}), \phi\left(\mathrm{X}^{\prime}\right), \phi\left(\mathrm{Y}^{\prime}\right)$ be an irreducible realization of these structure relations by skew-Hermitian operators on a Hilbert space $\mathrm{H} . \mathrm{X}^{2}+\mathrm{Y}^{\prime}{ }^{2}$ is a Casimir operator; we will normalize the representation $\phi$ so that

$$
\phi\left(\mathrm{X}^{\prime 2}+\mathrm{Y}^{2}\right)=-1
$$

Put

$$
\begin{aligned}
\mathcal{g}_{\lambda}(\mathrm{Z}) & =\phi(Z) \\
\rho_{\lambda}(\mathrm{Z}) & =\phi\left(\left[\mathrm{Z}^{2}, \mathrm{X}^{\prime}\right]+\lambda \mathrm{X}^{\prime}\right) \\
\mathcal{Q}_{\lambda^{\prime}}(\mathrm{Y}) & =1 / 2 \phi\left(\left[\mathrm{Z}^{2}, \mathrm{Y}^{\prime}\right]+\lambda \mathrm{Y}^{\prime}\right) \\
& -12-
\end{aligned}
$$


Then, these operators satisfy the structure relations for $\mathrm{SO}(3, \mathrm{R}),[12]$, Part I, page 260. Let us compute the value of the Casimir operator:

$$
\begin{aligned}
{\left[\mathrm{Z}^{2}, \mathrm{X}^{\prime}\right] } & =\mathrm{Z}^{\prime}+\mathrm{Y}^{\prime} \mathrm{Z} \\
& =2 \mathrm{Y}^{\prime} \mathrm{Z}-\mathrm{X}^{\prime},
\end{aligned}
$$

or

$$
\begin{aligned}
\rho_{\lambda}(X) & =\phi\left(X^{\prime}(\lambda-1 / 2)+Y^{\prime} Z\right) \\
\rho_{\lambda}\left(X^{2}\right) & =\phi\left(X^{\prime}{ }^{2}(\lambda-1 / 2)^{2}+Y^{\prime} Z Y^{\prime} Z+(\lambda-1 / 2)\left(X^{\prime} Y^{\prime} Z+Y^{\prime} Z X^{\prime}\right)\right. \\
& =\phi\left(X^{\prime}{ }^{2}(\lambda-1 / 2)^{2}+Y^{\prime 2} Z^{2}-Y^{\prime} X^{\prime} Z+(\lambda-1 / 2)\left(2 X^{\prime} Y^{\prime} Z+Y^{\prime}{ }^{2}\right)\right) \\
{\left[Z^{2}, Y^{\prime}\right] } & =-Z X^{\prime}-X X^{\prime} Z \\
& =-2 X^{\prime} Z-Y^{\prime}, \\
\rho_{\lambda}(Y) & =\phi\left(Y^{\prime}(\lambda-1 / 2)-X^{\prime} Z\right) \\
\left.\rho_{\lambda} Y^{2}\right) & =\phi\left(Y^{\prime}{ }^{2}(\lambda-1 / 2){ }^{2}+X^{\prime} Z X Z-(\lambda-1 / 2)\left(Y^{\prime} X^{\prime} Z+X^{\prime} Z Y^{\prime}\right)\right) \\
& =\phi\left(Y^{\prime}{ }^{2}(\lambda-1 / 2)^{2}+X^{2} Z^{2}+X^{\prime} Y^{\prime} Z-(\lambda-1 / 2)\left(2 Y^{\prime} X^{\prime} Z-X^{\prime}{ }^{2}\right)\right)
\end{aligned}
$$

Then,

$$
\begin{aligned}
\rho_{\lambda}\left(\mathrm{Z}^{2}+\mathrm{X}^{2}+\mathrm{Y}^{2}\right) & =-(\lambda-1 / 2)^{2}-(\lambda-1 / 2) \\
& =-(\lambda-1 / 2)(\lambda+1 / 2), \quad \text { or } \ell=\lambda-1 / 2
\end{aligned}
$$

Let $\psi_{j}, j=0, \pm 1, \pm 2, \ldots$, be a basis of $H$ such that

$$
\phi(\mathrm{Z}) \psi_{\mathrm{j}}=\mathrm{ij} \psi_{\mathrm{j}}
$$

Define:

$$
f_{l}^{j}(t)=\left\langle\psi_{o}\right| \rho_{\lambda+1 / 2}(\operatorname{Exp}(\mathrm{tX})) \psi_{\mathrm{j}}>
$$


This formula extends the "Legendre functions" defined by (4.1) to integral values of $j$ and arbitrary values of $\ell$. (To extend the definition to non-integral $j$, one must consider representation of the algebra $X^{\prime}, Y^{\prime}, Z$ in which $\phi(Z)$ has a non-discrete spectrum, a refinement we will not consider here.)

We get an asymptotic theorem for the Legendre function by transforming from the "Inonu-Wigner picture" (in which there is a singularity at $\lambda=\infty$ ) to the "Kodaira-Spencer picture" (in which a family of Lie algebras deforms as $\lambda \rightarrow \infty$ into the Lie algebra generated by $\left.Z, X^{\prime}, Y^{\prime}\right)$. In fact, define:

$$
\begin{aligned}
& \phi_{\lambda}(\mathrm{Z})=\phi(\mathrm{Z}) \\
& \phi_{\lambda}(\mathrm{X})=\frac{1}{\lambda} \rho_{\lambda}(\mathrm{X}) \\
& \phi_{\lambda}(\mathrm{Y})=\frac{1}{\lambda} \rho_{\lambda}(\mathrm{Y})
\end{aligned}
$$

As we already have verified $[11], \phi_{\lambda}$ is a representation of the following Lie algebra:

$$
[\mathrm{Z}, \mathrm{X}]_{\lambda}=\mathrm{Y},[\mathrm{Z}, \mathrm{Y}]_{\lambda}=-\mathrm{X}[\mathrm{X}, \mathrm{Y}]=\frac{\mathrm{Z}}{\lambda},
$$

which is a deformation of the Kodaira-Spencer sense of the Lie algebra generated by $Z, X^{\prime}, Y^{\prime}$.

In particular, everything is smooth as $\lambda \rightarrow \infty$, and

$$
\begin{aligned}
\lim _{\lambda \rightarrow \infty} & <\psi_{\mathrm{O}} \mid \phi_{\lambda}(\operatorname{Exp}(\mathrm{tx})) \psi_{\mathrm{j}}> \\
& =<\psi_{\mathrm{O}} \mid \phi\left(\operatorname{Exp}\left(\mathrm{tX} \mathrm{X}^{\prime}\right)\right) \psi_{\mathrm{j}}>
\end{aligned}
$$

The right hand side is just the matrix element for the given representation $\phi$ of the group of rigid motions of the plane. In fact, this is known to be $J_{j}(t)$, 
the Bessel function. The left hand side is

$$
\begin{aligned}
<\psi_{\mathrm{o}} \mid & \operatorname{Exp} \phi_{\lambda}(\mathrm{tX}) \psi_{\mathrm{j}}> \\
& =<\psi_{\mathrm{o}} \mid \operatorname{Exp}\left(\rho_{\lambda}\left(\frac{\mathrm{t}}{\lambda} \mathrm{X}\right)\right) \psi_{\mathrm{j}}> \\
& =\mathrm{f}_{\ell-1 / 2}^{\mathrm{j}}(\mathrm{t} / \lambda) .
\end{aligned}
$$

Hence

$$
\lim _{\ell \rightarrow \infty} f_{\ell}^{j}(t / \ell)=J_{j}(t)
$$

This is the asymptotic formula proved by Inonu and Wigner [13] by contracting (in their sense) a series of finite dimensional representations of $\mathrm{SO}(3, \mathrm{R})$. Note that our method, besides being more systematic, extends the formula automatically to nonintegral values of $\ell$ and $j$ and, if one is careful (as we have not been) to at least certain complex values of $t$. Another virtue of our method is that it extends routinely to representations of other groups to which the GellMann formula applies. Many such examples for semisimple groups are given in $[11]$.

We shall now turn to the physically more interesting case of the contraction of the Poincaré group to the Galilean group.

\section{CONTRACTION OF REPRESENTATIONS OF THE POINCARE' TO THE GALILEAN GROUP}

The topic of the title of this section is one of the standard examples illustrating the Inonu-Wigner ideas [12]. However, the interesting feature that "true" representations of the Poincaré group contract to ray representations of the Galilean 
group suggests that the physicist's way of looking at this be rephrased (for maximal clarity, if nothing else) in terms of our basic "analytic continuation" program. We have done this in [12], Part III, for the simplest sort of situation. However, we will now present it in simpler form that as a bonus, completely covers the general case! This way of looking at the problem is due to L. L. Foldy [4], and in this section we shall only be adapting his work to our point of view.

First, we must describe the deformation of the Poincare into the Galilean algebra. Let $\mathrm{G}$ be a vector space spanned by elements

$$
P_{i}, H, J_{i}, K_{i}
$$

$(\leq i, j, \ldots, \leq 3$ summation convention in force). Put $\lambda=1 / \mathrm{c}(\mathrm{c}=$ velocity of light). Define a one-parameter family of Lie algebra structures on $G$ as follows:

$$
\begin{aligned}
& {\left[P_{i}, P_{j}\right]_{\lambda} }=0=\left[P_{i}, H\right]_{\lambda}=\left[J_{j}, H\right]_{\lambda} \\
& {\left[J_{i}, J_{j}\right]_{\lambda}=-\epsilon_{i j k} J_{k}, } {\left[J_{i}, P_{j}\right]_{\lambda}=-\epsilon_{i j k} P_{k} } \\
& {\left[J_{i}, K_{j}\right]_{\lambda}=-\epsilon_{i j k} K_{k} } \\
& {\left[H, K_{j}\right]_{\lambda}=P_{j} } \\
& {\left[K_{i}, K_{j}\right]_{\lambda}=\lambda^{2} \epsilon_{i j k} J_{k} } \\
& {\left[P_{i}, K_{j}\right]_{\lambda}=\lambda^{2} \delta_{i j} H }
\end{aligned}
$$

$\left(\epsilon_{i j k}\right.$ is the usual skew-symmetric tensor). 
For $\lambda \neq 0$, this is the Lie algebra of the Poincare' group, for $\lambda=0$ that of the Galilean group. Notice that it is perfectly analytic in $\lambda$.

Now, we want to construct a Lie algebra $\mathrm{L}$, and a one-parameter family $\phi_{\lambda}$ of linear maps $G \rightarrow L$ which, for $\lambda \neq 0$, is a homomorphism of the $\lambda$-th structure into $\mathrm{L}$. For this, construct two Lie algebras $\underset{m}{H}$ and $\mathrm{S} . \mathrm{H}$ is an 8-dimensional Lie algebra, with basic elements $\left(p_{i}, x_{i}, 1, t\right)$ subject to the following commutation relations:

$$
\begin{aligned}
0=\left[p_{i}, p_{j}\right] & -\left[x_{i}, x_{j}\right]=\left[p_{i}, 1\right]=\left[x_{i}, 1\right] \\
& =\left[p_{i}, t\right]=\left[x_{i}, t\right] \\
{\left[p_{i}, x_{j}\right] } & =\delta_{i j} 1
\end{aligned}
$$

$\underline{\mathrm{S}}$ is a simple three-dimensional Lie algebra (whose Lie group is then $\operatorname{SO}(3, \mathrm{R})$ or $S U(2))$ generated by elements $\left(s_{i}\right)$ with

$$
\left[s_{i}, s_{j}\right]=-\epsilon_{i j k} s_{k}
$$

Now, let $\mathrm{L}=\mathrm{U}(\mathrm{H}+\mathrm{S})$, i. e., $\mathrm{H}+\mathrm{S}$ is the direct sum Lie algebra, and $U(H+S)$ is its universal enveloping algebra considered as a Lie algebra via the commutator. (We will in fact assume that $U(H+S)$ is the "complete" enveloping algebra, i.e., including arbitrary "functions" of the generators.)

Now, define $\phi_{\lambda}: G \rightarrow I_{s \neq t}$ as follows:

$$
\begin{aligned}
& \phi_{\lambda}\left(\mathrm{P}_{\mathrm{i}}\right)=\mathrm{p}_{\mathrm{i}} \\
& \phi_{\lambda}(\mathrm{H})=\epsilon \sqrt{\mathrm{m}^{2} \mathrm{c}^{2}+\mathrm{p}^{2}}, \mathrm{p}^{2}=\mathrm{p}_{\mathrm{i}} \mathrm{p}_{\mathrm{i}} \\
& \phi_{\lambda}\left(\mathrm{J}_{\mathrm{i}}\right)=\epsilon_{i j k} \mathrm{x}_{j} \mathrm{p}_{\mathrm{k}}+\mathrm{s}_{\mathrm{i}} \\
& \phi_{\lambda}\left(\mathrm{K}_{\mathrm{i}}\right)=\lambda^{2} \mathrm{x}_{\mathrm{i}} \phi_{\lambda}(\mathrm{H})-\epsilon_{i j k} \mathrm{~s}_{j} \mathrm{p}_{\mathrm{k}} /\left(\mathrm{mc} \mathrm{c}^{2}+\phi_{\lambda}(\mathrm{H})\right)-t \mathrm{p}_{i}
\end{aligned}
$$


One must now verify that $\phi_{\lambda}$ is a homomorphism of the $\lambda$-th Lie algebra defined by (5.1) into $I$. Let us take it for granted that this is done.

Of course, (5.2) is not analytic about $\lambda=0$, i. e., $c=\infty$. Let us see if the group theoretic trick introduced in [12], Part III, for "resolving the singularity," i.e., making a central extension, which is "trivial" for $\lambda \neq 0$ but nontrivial for $\lambda=0$, works again.

The trick to introduce an element 1 to $\mathrm{G}$ that commutes with all the generators of $\mathrm{G}$; for $\lambda \neq 0 . \phi_{\lambda}$ is also extended by mapping this element into the element 1 of $\underset{t=m}{H}$.

Consider:

$$
{ }^{\mathrm{r}}{ }_{\lambda}=\mathrm{H}-\mathrm{mc}^{2} \cdot 1=\mathrm{H}-\frac{\mathrm{m}^{2} \cdot 1}{\lambda^{2}}
$$

Then, all the relations (5.1) are unchanged by this substitution of $\mathrm{H}_{\lambda}^{\prime}$ for $\mathrm{H}$, except possibly the last:

$$
\left[\mathrm{P}_{\mathrm{i}}, \mathrm{K}_{\mathrm{j}}\right]=\lambda^{2} \delta_{i j} \mathrm{H}=\lambda^{2} \delta_{\mathrm{ij}}\left(\mathrm{H}_{\lambda}+\frac{\mathrm{m} \cdot 1}{\lambda^{2}}\right)=\delta_{\mathrm{ij}}\left(\lambda^{2} \mathrm{H}_{\lambda}+\mathrm{m} \cdot 1\right)
$$

Notice now that the "lucky" accident mentioned in [12], Part III, repeats itself: The commutation relations do not pick up a singularity as $\lambda \rightarrow 0$. However, again this is at the expense of not getting the Galilean algebra itself as $\lambda \rightarrow 0$ but a central extension of it.

Finally, we must check that the homomorphism $\phi_{\lambda}$ also is analytic at $\lambda=0$. Let us look at (5.2). The only terms that are effected are the second 
and the fourth:

$$
\begin{aligned}
\phi_{\lambda}\left(\mathrm{H}_{\lambda}{ }^{\prime}\right) & =\phi_{\lambda}(\mathrm{H})-m c^{2} 1 \\
& =m c^{2} \sqrt{1+\frac{p^{2}}{m^{2} c^{2}}-m c^{2}} \\
= & m c^{2}+\frac{1}{2} \frac{p^{2}}{m}+\ldots-m c^{2} \\
= & \frac{1}{2} \frac{p^{2}}{m}+\ldots \\
\phi_{\lambda}\left(\mathrm{K}_{\mathrm{i}}\right)= & \lambda^{2} \mathrm{x}_{\mathrm{i}}\left(\phi_{\lambda}\left(\mathrm{H}_{\lambda}\right)+\frac{\mathrm{m}}{\lambda^{2}} 1\right) \\
& -\epsilon_{\mathrm{ijk}} \mathrm{s}_{\mathrm{j}} \mathrm{p}_{\mathrm{k}} /\left(\frac{\mathrm{m}}{\lambda^{2}}+\phi_{\lambda}\left(\mathrm{H}_{\lambda}\right)+\frac{\mathrm{m}}{\lambda^{2}}-1\right) \\
& -\mathrm{tp}_{\mathrm{i}}
\end{aligned}
$$

Again, we see that there is no trouble at $\lambda=0$ : The representation $\phi_{\lambda}$ is now analytic at $\lambda=0$. We conclude that Foldy's formalism gives an excellent description of the simultaneous "deformation" or "analytic continuation" of the Poincaré algebra and its representations into those of the Galilean algebra. 


\section{ANALYTIC CONTINUATION OF THE PLANCHEREL FORMULA FOR $\mathrm{SO}(2, \mathrm{R})^{*}$}

Before studying "analytic continuation" of matrix elements in generality, it will be useful to examine some simple examples. $S O(2, R)$ seems to be the simplest case that gives some insight into the general case.

Consider two complex variables $\mathrm{x}$ and $\mathrm{y}$, and the quadric $\mathrm{M}$ defined in this space by

$$
x^{2}+y^{2}=1
$$

$M$ is, of course, just the group manifold of $\mathrm{SO}(2, \mathrm{C})$. Let $\mathrm{M}^{\mathrm{O}}$ be the points of $\mathrm{M}$ for which $\mathrm{x}$ and $\mathrm{y}$ are real, and let $\mathrm{M}^{\top}$ be the points for which $\mathrm{x}$ is real, while $y$ is pure imaginary. $M^{O}$ is the group manifold of $S O(2, R), M^{\prime}$ the group manifold of $S O(1,1)$. Both $\mathrm{M}^{\mathrm{O}}$ and $\mathrm{M}^{\prime}$ are "real forms" of $\mathrm{M}$, with $M^{\circ}$ compact (i.e., a circle) while $M^{\prime}$ is non-compact (i.e., a hyperbola).

Suppose $f(x, y)$ is a function defined on $M$ alone, analytic in a suitable domain, continuous when restricted to $\mathrm{M}^{\mathrm{O}}$, with boundary values (possibly in a generalized sense) on $\mathrm{M}^{\prime}$. Now, f restricted to $\mathrm{M}^{\circ}$ can be expanded in a Fourier series (which is then the Peter-Weyl expansion for $\mathrm{SO}(2, \mathrm{R})$ ) while the boundary values of $f(x, y)$ on $M$ can be expanded in a Fourier integral (which is the Plancherel expansion for the group $\mathrm{SO}(1,1))$.

Our main task is to examine how the two expressions may be related to one another. Specifically, we have in mind developing a group-theoretic version of the Sommerfeld-Watson transform, i.e., some procedure for "analytically continuing" an expansion over a compact group into an expansion over a noncompact real form.

\footnotetext{
"I would lilse to thank A. Kihlberg who discovered an error in the first version of this section.
} 
Introduce a new variable $\mathrm{z}=\mathrm{x}+\mathrm{iy}$. Then the $\mathrm{z}$-plane minus the point $z=0$ represents $M$ as a complex manifold. $M^{\circ}$ is the set of $z$ of the form $e^{i \theta}, 0 \leq \theta \leq 2 \pi$, while $M^{\prime}$ is the real axis.

Coinsider the Fourier expansion of $f$ restricted to $\mathrm{M}^{\circ}$, i.e.,

$$
\begin{aligned}
& f\left(e^{i \theta}\right) \sim \sum_{n} f_{n} e^{i n \theta}, \\
& f_{n}=\frac{1}{2 \pi} \int_{0}^{2 \pi} f\left(e^{i \theta}\right) e^{-i n \theta} d \theta
\end{aligned}
$$

We must somehow take into account the fact that $f(z)$ is analytically continuable off the circle $|z|=1$, leading to "boundary values" on $M$ '. The simplest way of doing this can be described as follows:

Suppose $f(z)$ satisfies a "dispersion relation," in the form of a Cauchy integral representation:

$$
\begin{aligned}
& f(z)=\frac{1}{2 \pi i} \int_{1+\epsilon} \frac{f(t)}{t-z} d t \\
& \text { for some } \epsilon>0 .
\end{aligned}
$$

Use 6.2 and 6.1 , assuming that the integrals can be interchanged:

$$
\begin{aligned}
f n & =\frac{1}{2 \pi i} \int_{1+\epsilon}^{\infty}\left(\int_{0}^{2 \pi} \frac{e^{i n \theta}}{t-e^{i \theta}} d \theta\right) f(t) d t \\
& =\frac{1}{2 \pi i} \int_{1+\epsilon}^{\infty} f(t) t^{-n-1} d t \text { for } n \geq 0 \\
& =0 \text { for } n<0 .
\end{aligned}
$$

I owe this suggestion to A. Martin. 
Suppose $g(z)$ is a similar function, admitting a dispersion relation of the form:

$$
g(z)=\frac{1}{2 \pi i} \int_{\epsilon}^{1-\epsilon} \frac{g(t)}{t-z} d t
$$

Similarly,

$$
\begin{aligned}
g_{n} & =\frac{1}{4 \pi^{2} i} \int_{\epsilon}^{1-\epsilon}\left(\int_{0}^{2 \pi} \frac{e^{-i n \theta}}{t-e^{i \theta}} d \theta\right) g(t) d t \\
& =0 \text { if } n \geq 0 \\
& =\frac{1}{2 \pi i} \int_{\epsilon}^{1-\epsilon} t^{-n-1} g(t) d t \text { for } n<0
\end{aligned}
$$

Now, let $t=e^{S}$ in each of these integrals:

$$
\begin{aligned}
& f_{n}=\frac{1}{2 \pi i} \int_{\log (1+\epsilon)}^{\infty} f\left(e^{s}\right) e^{-n s} d s \\
& g_{n}=\frac{1}{2 \pi i} \int_{\log \epsilon}^{\log (1-\epsilon)} g\left(e^{s}\right) e^{-n s} d s
\end{aligned}
$$

As $\epsilon \rightarrow 0$, we obtain, in formal way at least,

$$
(f+g)_{n}=\frac{1}{2 \pi i} \int_{-\infty}^{\infty}(f+g)\left(e^{s}\right) e^{-n s} d s
$$

(Regard the function $(f+g)\left(e^{s}\right)$, defined as follows on the "boundary values" of the function $(f+g)\left(e^{i s}\right)$ on the non-compact set $M^{\prime}$ : 


$$
\begin{aligned}
& (f+g)\left(e^{s}\right)=f\left(e^{s}\right) \text { for } s>0 \\
& \left.(f+g)\left(e^{s}\right)=g\left(e^{s}\right) \text { for } s<0 .\right)
\end{aligned}
$$

So far, $\mathrm{n}$ has been real; however, 6.6 suggests that we use the formula for $\mathrm{n}$ pure imaginary as well, obtaining in this formal way, essentially the "Fourier transform" of the function $s \rightarrow(f+g)\left(e^{s}\right)$, i.e., the "Plancherel expansion" of this function when it is considered as a function on $\mathrm{SO}(1,1)$.

This simple explicit calculation immediately suggests a general framework. Suppose $\mathrm{G}$ is a non-compact semisimple Lie group. Let $\mathrm{G}_{\mathrm{c}}$ be its complexification, $G_{\mu}$ a compact subgroup of $G_{c}$ whose compactification is also $G_{c} \cdot\left(G_{\mu}\right.$ is the "compact real form" of $\left.G_{c}\right) . G_{\mu}$ can be chosen so that $G_{\mu} \cap G=K$ is a maximal compact subgroup of $\mathrm{G}$, and a symmetric subgroup of $\mathrm{G}_{\mu}$. (One class of examples to keep in mind might be:

$$
\begin{aligned}
& \mathrm{G}=\mathrm{SO}(\mathrm{n}, 1), \mathrm{G}_{\mathrm{c}}=\mathrm{SO}(\mathrm{n}+1, \mathrm{C}) \\
& \left.\mathrm{G}_{\mu}=\mathrm{SO}(\mathrm{n}+1, \mathrm{R}), \mathrm{K}=\mathrm{SO}(\mathrm{n}, \mathrm{R})\right)
\end{aligned}
$$

Let $K_{c}$ be the complexification of $K$, considered as a complex subgroup of $\mathrm{G}_{\mathrm{c}} \cdot$ Define the following coset spaces.

$$
\mathrm{M}=\mathrm{G}_{\mathrm{c}} / \mathrm{K}_{\mathrm{c}} ; \mathrm{M}^{\mathrm{O}}=\mathrm{G}_{\mu} / \mathrm{K} ; \mathrm{M}^{\prime}=\mathrm{G} / \mathrm{K}
$$

Then, both $\mathrm{M}^{\mathrm{O}}$ and $\mathrm{M}^{\prime}$ are "real" submanifolds of the comples manifold $M$, i.e., complex analytic coordinate systems can be found for MI for which, locally, each of $\mathrm{M}^{\mathrm{O}}$ and $\mathrm{M}^{\prime}$ are defined by real values of the coordinates. Denote points of $M, M^{0}, M^{\prime}$ by $p, p^{0}, p^{\prime}$, respectively. Suppose $f(p)$ is a function which is complex analytic on at least some domain of $\mathrm{M}$, and defined 
and continuous of $\mathrm{M}^{\circ}$. Then, $f\left(\mathrm{p}^{\circ}\right)$ can be expanded in a "Fourier series" using the spherical functions [10] of the symmetric space $G_{\mu} / K$. Let $\left\{f_{n}\right\}$ denote the coefficient in such an expansion ( $\mathrm{n}$ is a descrete, multiparameter index). Then

$$
f_{n}=\int_{M^{0}} f\left(p^{o}\right) h_{n}\left(p^{o}\right) d p^{o}
$$

where $h_{n}\left(p^{0}\right)$ is the spherical function parameterized by $n$, and $d p^{0}$ denotes a volume element on $\mathrm{M}^{\mathrm{O}}$ invariant under $\mathrm{G}_{\mu}$.

Now, $\mathrm{M}^{0}$ will intersect $\mathrm{M}^{\prime}$. Suppose, as $\epsilon \rightarrow 0, \mathrm{M}^{\prime}-\mathrm{M}^{\circ}$ can be written as the union of connected submanifolds $M_{\epsilon}^{1} \cup M_{\epsilon}^{2} U$... of the same dimension as $M^{\prime}$. (Precisely, we mean that, as $\epsilon \rightarrow 0$, this union fills up $M^{\prime}-M^{0}$.) Suppose $B\left(p^{o}, p^{\prime}\right)$ is a Cauchy (i.e., Bergman-Weil) integral kernel which converts a function $f\left(p^{\prime}\right)$ on $\mathrm{MI}^{\prime}$ into a holomorphic function $f(p)$ by the formula:

$$
f(p)=\int_{M^{\prime}} B\left(p^{\circ}, p^{\prime}\right) f\left(p^{\prime}\right) d p^{\prime},
$$

where $d p^{\prime}$ is a volume element on $M^{\prime}$ which is invariant under $G^{\prime}$. (We would also suppose that the operator $f\left(p^{\prime}\right) \rightarrow f(p)$ is an intertwining operator for the action of $G^{\prime}$ on these functions.) Suppose that, for $f^{j}\left(p^{\prime}\right)$ defined and continuous on $M_{\epsilon}^{j}, j=1,2, \ldots$ the formula:

$$
f^{j}\left(p^{o}\right)=\int_{M_{\epsilon}^{j}} B\left(p^{o}, p^{\prime}\right) f^{j}\left(p^{\prime}\right) d p^{\prime}
$$

gives a continuous function on $\mathrm{M}^{\circ}$. We can now obviously combine 6.8 with 6.7 to obtain:

$$
\mathrm{f}_{n}^{j}=\int_{M_{\epsilon}^{1}}\left(\int_{M^{O}} B\left(p^{o}, p^{\prime}\right) g_{n}\left(p^{o}\right) d p^{o}\right) f\left(p^{\prime}\right) d p^{\prime}
$$


which should be the generalization of 6.6. In particular, the functions:

$$
g_{n}(p)=\int_{M^{\circ}} B\left(p^{\circ}, p^{\prime}\right) g_{n}\left(p^{o}\right) d p^{0}
$$

should be the generalization of the "Legendre functions of the second kind," i.e., the functions that are, in the classical notation, $\mathrm{p}_{\mathrm{n}}(\cos \theta)$.

Since working further would involve considerably more complicated machinery, we shall leave these ideas as conjectures to be worked on at a later point. 


\section{GENERALIZED FUNCTIONS AND THE DIRAC NOTATIONS}

So far, in studying matrix elements $\mathrm{g} \rightarrow\left\langle\psi^{\prime}|\rho(\mathrm{g})| \psi\right\rangle$ of group representations we have assumed that $\psi$ and $\psi^{r}$ wore elements of a Hilbert space II, and that $\rho$ is a representation of a Lie group G by genuine operators mapping $\mathrm{H}$ into $\mathrm{H}$. While this is adequate for simple problems, clearly many of the interesting applications to physics involve such objects defined when $\psi$ and $\psi^{\prime}$ are "generalized functions" in the sense uscd by Gclfand and his coworkers. We will now present some of these ideas in a form that will be most useful for our goal of relating group representation theory to clcmentary particle physics and quantum field theory.

It is interesting to note that Gelfand and Valenkin's version of the theory [9] is extremely close to that originally used by Dirac in his treatment of quantum mechanics, although they avoid Dirac's ingenious notations, presumably because of its bad reputation among mathematicians. In our brief treatment here, we will attempt to suggest such a direct use of Dirac's ideas, although since it is only a side point, we cannot claim that all the details have been fully developed.

Now we proceed to the description of the notations and ideas we will need. A Hilbert space will be, in this paper, a complex vector space, elements typically denoted by $\psi, \psi^{\prime}, \ldots$, with a positive-definite Hermitian inner product $\left(\psi, \psi^{\prime}\right) \rightarrow<\psi \mid \psi^{\dagger}>$, which satisfies:

$$
\begin{aligned}
& <\psi\left|\psi^{\prime}\right\rangle=\left\langle\psi^{\prime} \mid \psi\right\rangle^{*} \quad(* \text { denotes complex conjugate) } \\
& <\mathrm{c} \psi\left|\psi^{\prime}\right\rangle=\mathrm{c}^{*}\left\langle\psi \mid \psi^{\prime}\right\rangle \text { for } \mathrm{c} \in \mathrm{C} \quad \text { (C denotes the complex numbers) } \\
& <\psi\left|\mathrm{c} \psi^{\prime}\right\rangle=\mathrm{c}\left\langle\psi \mid \psi^{\prime}\right\rangle
\end{aligned}
$$


We will not assume that $\mathrm{H}$ is complete, as is customary in most work in functional analysis.

Let $\mathrm{D}$ be a complex-vector space of linear functionals on $\mathrm{H}$. For intuitive purposes, we will denote elements of $\mathrm{D}$ and $\mathrm{H}$ by the same sort of letter, usually $\psi$. For $\psi \in D, \psi^{\prime} \in \mathrm{H},\left\langle\psi \mid \psi^{\prime}\right\rangle$ is the value of the linear functional on $\psi \prime$ '. We will suppose that:

(a) D is a topological vector space. (Datails about the topology of D will be made precise as they are needed. Recall that Gelfand and Valenkin define $\mathrm{D}$ as the dual space, with standard dual space topology, of a topology on $\mathrm{H}$ that is different from the topology on $\mathrm{H}$ defined by the norm $\|\psi\|=\sqrt{\langle\psi \mid \psi\rangle}$.)

(b) For fixed $\psi_{\mathrm{o}} \in \mathrm{H}$, the linear form $\psi^{\prime} \rightarrow\left\langle\psi_{\mathrm{o}}\right| \psi^{\prime}>$ is in D. This serves to identify $\mathrm{H}$ with a subspace of $\mathrm{D}$. When we write $\mathrm{HCD}$, this is what we mean. $\mathrm{D}$ will be called the Dirac space associated with the Hilbert space $\mathrm{H}$,

(c) Any linear form in $\mathrm{H}$ that is continuous with respect to the Hilbert space topology defined by the norm $\|\psi\|$ is also in D. (Thus, D contains the completion of $\mathrm{H}$ as well as $\mathrm{H}$ itself.)

Note that $\left\langle\psi \mid \psi^{\prime}\right\rangle$ does not make sense as a number if neither $\psi$ nor $\psi^{\prime}$ belongs to $H$. However, it can be given a meaning by a limiting process, or regarded merely as a convenient algebraic symbol in certain specific calculations. To see this, we consider the idea of a "generalized orthonormal basis" of $H$, which we now define.

Let $M$ be an auxiliary space, whose points are denoted by $p, p^{\prime}, q$, etc. Let $d p$ be a measure on $M$, enabling one to form the integral $\int \hat{I}(p) d p$ of a 
of a certain class of complex-valued functions on $M$. Consider a mapping $\mathrm{M} \rightarrow \mathrm{D}$, denoted by $\mathrm{p} \rightarrow \psi_{\mathrm{p}}$. It is said to form an orthonormal basis for $\mathrm{H}$ if

$$
<\psi \mid \psi^{\prime}>=\int\left\langle\psi\left|\psi_{\mathrm{p}}><\psi_{\mathrm{p}}\right| \psi^{\prime}>\mathrm{dp}\right.
$$

$$
\text { for } \psi, \psi^{\prime} \in \mathrm{H}
$$

This relation can be symbolized by the relation:

$$
<\psi_{\mathrm{p}} \mid \psi_{\mathrm{q}}>=\delta_{\mathrm{p}, \mathrm{q}} \quad \text { for } \mathrm{p}, \mathrm{q} \in \mathrm{M}
$$

Now, suppose that $A$ is a linear operator: $\mathrm{H} \rightarrow \mathrm{H}$, define $A^{*}: D \rightarrow D$ by the formula

$$
<\mathrm{A}^{*} \psi\left|\psi^{\prime}\right\rangle=\left\langle\psi \mid \mathrm{A} \psi^{\prime}\right\rangle \quad \text { for } \psi \in \mathrm{D}, \psi^{\prime} \in \mathrm{H} \text {. }
$$

The operators A that we will consider will satisfy the following condition:

$$
A^{*}(\mathrm{H}) \subset \mathrm{H}
$$

Then, we can extend $A$ to a map: $D \rightarrow D$ by the formula

$$
<\mathrm{A} \psi\left|\psi^{\prime}\right\rangle=\left\langle\psi \mid \mathrm{A} * \psi^{\prime}\right\rangle \text { for } \psi^{\prime} \in \mathrm{H}, \psi \in \mathrm{D}
$$

A vector $\psi \in \mathrm{D}$ is then an eigenvector for $A$ with eigenvalue $\lambda$ if

$$
\mathrm{A} \psi=\lambda \psi
$$

$\mathrm{H}$ is diagonal with respect to the orthonormal basis $\mathrm{p} \rightarrow \psi_{\mathrm{p}}$ if

$$
A \psi_{p}=\lambda(p) \psi_{p} \text { for all } p \in M
$$

Several examples will make the ideas clear:

Example 1 - The Dirac Delta Function

Suppose that $M$ is a locally compact topological space*, and the measure $\mathrm{dp}^{*}$ is such that all compact sets are measurable and have finite measure.

\footnotetext{
* It would suffice to think of Euclidean space $R^{\mathrm{n}}$ as an example, with the Euclidean measure $d p=d x^{n}$.
} 
(dp is then sometimes called a Radon measure.) For $H$ we can takc the space of all continuous, complex-valued functions $\mathrm{p} \rightarrow \psi(\mathrm{p})$ on $\mathrm{M}$ that vanish outside of a compact set with $\left\langle\psi \mid \psi^{\prime}\right\rangle=\int \psi(\mathrm{p})^{*} \psi^{\prime}(\mathrm{p}) \mathrm{dp}$. For $\mathrm{p} \in \mathrm{M}$, define $\delta_{\mathrm{p}} \in \mathrm{V}$ as follows:

$$
\delta_{\mathrm{p}}(\psi)=\psi(\mathrm{p})=\left\langle\delta_{\mathrm{p}}\right| \psi>\text { for } \psi \epsilon \mathrm{H}
$$

Then, for $\psi, \psi^{\prime} \in \mathrm{H}$,

$$
\begin{aligned}
\int<\left.\psi\right|_{\mathrm{p}}><\delta_{\mathrm{p}} \mid \psi^{\prime}>\mathrm{dp} & =\int<\delta_{\mathrm{p}}|\psi\rangle^{*}<\delta_{\mathrm{p}}\left|\psi^{\prime}\right\rangle \mathrm{dp} \\
& =\psi(\mathrm{p})^{*} \psi^{\prime}(\mathrm{p}) \mathrm{dp}=\left\langle\psi \mid \psi^{\prime}\right\rangle
\end{aligned}
$$

i. e., (7. 1) is satisfied.

Let $A$ be the operation of multiplication by a continuous, complex valued function $\mathrm{p} \rightarrow \mathrm{f}(\mathrm{p})$ on $\mathrm{M}$. Then, $(\mathrm{A} \psi)(\mathrm{p})=\mathrm{f}(\mathrm{p}) \psi(\mathrm{p})$.

$A^{*}$, on $H$, is obviously just the action of multiplication by $f^{*}$, hence maps $\mathrm{H}$ into itself. Then

$$
\begin{aligned}
\left\langle\mathrm{A} \delta_{\mathrm{p}} \mid \psi\right\rangle & =\left\langle\delta_{\mathrm{p}} \mid \mathrm{A}^{*} \psi\right\rangle=\left\langle\delta_{\mathrm{p}} \mid \mathrm{f} * \psi\right\rangle \\
& =\mathrm{f}(\mathrm{p})^{*} \psi(\mathrm{p})=\left\langle\mathrm{f}(\mathrm{p}) \delta_{\mathrm{p}} \mid \psi\right\rangle
\end{aligned}
$$

i. e., $\delta_{\mathrm{p}}$ is an eigenvector for $\mathrm{A}$.

Example 2- The Fourier Integral

Let $\mathrm{x}$ denote a real variable, $-\infty<\mathrm{x}<\infty$, and let $\mathrm{H}$ be the space of infinitely differentiable functions $x \rightarrow \psi(x)$ that vanish outside of a compact set.

$$
\left\langle\psi \mid \psi^{\prime}\right\rangle=\int_{-\infty}^{\infty} \psi(x)^{*} \psi^{\prime}(\mathrm{x}) \mathrm{dx}
$$


Let $\mathrm{p}$ also be a real variable, $-\infty<\mathrm{p}<\infty$, and for each $\mathrm{p} \in \mathrm{M}$, define $\psi_{\mathrm{p}} \in \mathrm{D}$ by the formula:

$$
\left\langle\psi_{\mathrm{p}} \mid \psi\right\rangle=\frac{1}{\sqrt{2 \pi}} \int_{-\infty}^{\infty} \mathrm{e}^{-\mathrm{ipx}} \psi(\mathrm{x}) \mathrm{dx}
$$

Notice that $\left\langle\psi_{\mathrm{p}} \mid \psi\right\rangle=\hat{\psi}(\mathrm{p})$, where $\hat{\psi}$ denotes the Fourier transform of $\psi$. Then

$$
\begin{aligned}
\int_{-\infty}^{\infty}\left\langle\psi \mid \psi_{\mathrm{p}}\right\rangle\left\langle\psi_{\mathrm{p}} \mid \psi^{\prime}\right\rangle \mathrm{dp} & =\int_{-\infty}^{\infty}\left\langle\psi_{\mathrm{p}}\right| \psi^{*}\left\langle\psi \mid \psi^{\prime}\right\rangle \mathrm{dp} \\
& =\int_{-\infty}^{\infty} \hat{\psi}(\mathrm{p})^{*} \hat{\psi}(\mathrm{p}) \mathrm{dp}=\left\langle\psi \mid \psi^{\prime}\right\rangle
\end{aligned}
$$

This is usually called the "Plancherel formula," and expresses the fact that the Fourier transform is a unitary operator. We see that it is equivalent to the statement that the $\psi_{\mathrm{p}}$ form on orthonormal basis. (If one requires

$$
\psi=\int_{-\infty}^{\infty}<\psi \mid \psi_{\mathrm{p}}>\psi_{\mathrm{p}} \mathrm{dp}
$$

where the integral on the right-hand side is obtained as a limit of partial sums, using another topology in D, then one finds equivalent statements to the Fourier inversion formulas.)

Define $\mathrm{A}$ as the operator $\psi(\mathrm{x}) \rightarrow \mathrm{d} / \mathrm{dx} \psi(\mathrm{x})$. Then it is readily computed that $A^{*}=-d / d x$, and maps $H$ into itself.

$$
\begin{aligned}
\left\langle\mathrm{A} \psi_{\mathrm{p}} \mid \psi\right\rangle & =\left\langle\psi_{\mathrm{p}} \mid \mathrm{A}^{*} \psi\right\rangle=\frac{1}{\sqrt{2 \pi}} \int_{-\infty}^{\infty}-\mathrm{e}^{-\mathrm{ipx}} \mathrm{d} / \mathrm{dx} \psi(\mathrm{x}) \mathrm{dx} \\
& =-\frac{1}{\sqrt{2 \pi}} \operatorname{ip}_{-\infty}^{\infty} \mathrm{e}^{-\mathrm{ipx}} \psi(\mathrm{x}) \mathrm{dx} \\
& =-\operatorname{ip}\left\langle\psi_{\mathrm{p}}\right| \psi>=\left\langle\mathrm{ip} \psi_{\mathrm{p}} \mid \psi\right\rangle \\
& -30-
\end{aligned}
$$


This is interpreted as saying that $\psi_{\mathrm{p}}$ is an eigenvector for $\mathrm{A}$, with eigenvalue ip.

We now turn from these trivial examples to a situation where in addition a group acts. Let $\mathrm{H}, \mathrm{D}$ and $\mathrm{M}$ be as above. Suppose $\mathrm{p} \rightarrow \psi_{\mathrm{p}}$ defines an orthonormal basis for $\mathrm{H}$. In addition, suppose that $\mathrm{G}$ is a group, and that $\rho: \mathrm{g} \rightarrow \rho(\mathrm{g})$ is a representation of $\mathrm{G}$ by operators on H. Suppose $\rho(\mathrm{g})$ * maps $\mathrm{H}$ into itself, so that $\rho(g)$ can be extended to an operator: $\mathrm{D} \rightarrow \mathrm{D}$.

Definition - The orthonormal basis $\mathrm{p} \rightarrow \psi_{\mathrm{p}}$ of $\mathrm{H}$ is compatible with the action of $G$ on $H$ if:

(a) $G$ acts as a transformation group on $M$ : For $p \in M, g \in G$, gp denotes the transform of $\mathrm{p}$ by $\mathrm{g}$.

(b) $\quad \rho(g)\left(\psi_{\mathrm{p}}\right)=\mathrm{m}(\mathrm{g}: \mathrm{p}) \psi_{\mathrm{gp}}$ for $\mathrm{g} \in \mathrm{G}, \mathrm{p} \in \mathbb{M}$.

The map $(\mathrm{g}, \mathrm{p}) \rightarrow \mathrm{m}(\mathrm{g}, \mathrm{p})$ is a complex-valued function on $\mathrm{G} \times \mathrm{M}$ that is called the multiplier system associated with the orthonormal basis and the group action.

$m$ cannot be an arbitrary function, but must satisfy a certain functional equation. In fact,

$$
\begin{aligned}
\rho\left(\mathrm{g}_{1} \mathrm{~g}_{2}\right)\left(\psi_{\mathrm{p}}\right) & =\mathrm{m}\left(\mathrm{g}_{1} \mathrm{~g}_{2}, \mathrm{p}\right) \psi_{\mathrm{g}_{1} \mathrm{~g}_{2} \mathrm{p}} \\
& =\rho\left(\mathrm{g}_{1}\right)\left(\rho\left(\mathrm{g}_{2}\right) \psi_{\mathrm{p}}\right) \\
& =\rho\left(\mathrm{g}_{1}\right)\left(\mathrm{m}\left(\mathrm{g}_{2}, \mathrm{p}\right) \psi_{\mathrm{g}_{2} \mathrm{p}}\right) \\
& =\mathrm{m}\left(\mathrm{g}_{2}, \mathrm{p}\right) \mathrm{m}\left(\mathrm{g}_{1}, \mathrm{~g}_{2} \mathrm{p}\right) \psi_{\mathrm{g}_{1} \mathrm{~g}_{2} \mathrm{p}}
\end{aligned}
$$

or

$$
\mathrm{m}\left(\mathrm{g}_{1} \mathrm{~g}_{2}, \mathrm{p}\right)=\mathrm{m}\left(\mathrm{g}_{2}, \mathrm{p}\right) \mathrm{m}\left(\mathrm{g}_{1}, \mathrm{~g}_{2} \mathrm{p}\right)
$$

This is called the multiplier equation. We can rephrase its basic property in the following way: 
Theorem 7.1 Suppose $\mathrm{p} \rightarrow \psi_{\mathrm{p}}$ is an orthonormal basis for $\mathrm{H}$, and that $\mathrm{m}(\mathrm{g}, \mathrm{p})$ satisfies the multiplier Eq. (7.3). Let $\mathrm{g} \rightarrow \rho^{\prime}(\mathrm{g})$ be a representation of $G$ by linear transformation on $V$, such that $\rho^{\prime}(g)\left(\psi_{\mathrm{p}}\right)$ is a multiple of $\psi_{\mathrm{gp}}$. For $\mathrm{g} \epsilon \mathrm{G}$, define $\rho(\mathrm{g})$ as a linear transformation on $\mathrm{D}$ by the formula:

$$
\rho(\mathrm{g})\left(\psi_{\mathrm{p}}\right)=\mathrm{m}(\mathrm{g}, \mathrm{p}) \rho^{\prime}(\mathrm{g})\left(\psi_{\mathrm{p}}\right)
$$

Then, $\rho \mathrm{g} \rightarrow \rho(\mathrm{g})$ also is a representation of $\mathrm{G}$ by linear transformations on $\mathrm{V}$.

Proof:

$$
\begin{aligned}
\rho\left(g_{1} g_{2}\right)\left(\psi_{\mathrm{p}}\right) & =m\left(g_{1} g_{2}, p\right) \rho^{\prime}\left(g_{1} g_{2}\right)\left(\psi_{p}\right) \\
& =m\left(g_{2}, p\right) m\left(g_{1}, g_{2} p\right) \rho^{\prime}\left(g_{1}\right) \rho^{\prime}\left(g_{2}\right)\left(\psi_{p}\right) \\
& =m\left(g_{1}, g_{2} p\right) \rho^{\prime}\left(g_{1}\right)\left(m\left(g_{2}, p\right) \rho^{\prime}\left(g_{2}\right)\left(\psi_{p}\right)\right) \\
& =m\left(g_{1}, g_{2} p\right) \rho^{\prime}\left(g_{1}\right)\left(\rho\left(g_{2}\right)\left(\psi_{p}\right)\right) \\
& =\rho\left(g_{1}\right) \rho\left(g_{2}\right)\left(\psi_{p}\right), \text { i.e. } \\
\rho\left(g_{1} g_{2}\right) & =\rho\left(g_{1}\right) \rho\left(g_{2}\right) . \quad \text { q.e.d. }
\end{aligned}
$$

Theorem 7.2 If $\mathrm{m}\left(\mathrm{g}_{1} \mathrm{p}\right)$ satisfies the multiplier equation, so does any power $m\left(g_{1}, p\right)^{a}$, where a is a complex number.

Proof: This is obvious from 7.3 .

Now fix a point $q \in M$, and consider the isotropy subgroup of $G$ at $q$, that we denote by $\mathrm{G}^{\mathrm{q}}$

$$
G^{q}=\{g \in G: g q=q\}
$$


Theorem 7.3 If $\mathrm{m}(\mathrm{g}, \mathrm{p})$ is a multiplier system, then the mapping $\mathrm{g} \rightarrow \mathrm{m}\left(\mathrm{g}, \mathrm{p}_{\mathrm{o}}\right)$ is, for $\mathrm{g} \in \mathrm{G}^{\mathrm{p}_{\mathrm{o}}}$, a homomorphism of $\mathrm{G}^{\mathrm{q}}$ into the complex numbers.

Proof: For $\mathrm{G}_{1}, \mathrm{~g}_{2} \in \mathrm{G}^{\mathrm{q}}$,

$$
\begin{aligned}
\mathrm{m}\left(\mathrm{g}_{1} \mathrm{~g}_{2}, \mathrm{q}_{\mathrm{o}}\right) & =\mathrm{m}\left(\mathrm{g}_{2}, q\right) \mathrm{m}\left(\mathrm{g}_{1}, \mathrm{~g}_{2} q\right) \\
& =\mathrm{m}\left(\mathrm{g}_{2}, q\right) \mathrm{m}\left(\mathrm{g}_{1}, q\right)
\end{aligned}
$$

which exhibits the homomorphism property.

As is well-known, this formalism applies to describe the spin-zero representations of the connected Poincare'group, $G=L T$, where $L$ is the subgroup of Lorentz rotations, $T$ the invariant subgroup of translations. $M$ is the "mass-shell positive energy" subset of the vector space of 4-momenta, that we denote by $R^{2}$, i.e.,

$$
M=p \in R^{4}: p \cdot p=m^{2}, p_{O}>0
$$

$(\mathrm{p} \cdot \mathrm{p}$ denotes the Lorentz inner product)

If

$$
p=\left(p^{0}, p^{1}, p^{2}, p^{3}\right) \text { then } p \cdot p=\left(p^{o}\right)^{2}-\left(p^{1}\right)^{2}-\left(p^{2}\right)^{2}-\left(p^{3}\right)^{2}
$$

The elements $\psi_{\mathrm{p}}$ for $\mathrm{p} \in \mathrm{M}$, are then the $\delta$-functions in momenta.

$$
\rho(\ell) \psi_{\mathrm{p}}=\psi_{\ell \mathrm{p}} \text { for } \quad \ell \in \mathrm{L}, \mathrm{p} \in \mathrm{M}, \quad \text { i.e. }
$$

the action of $\mathrm{L}$ is just that of the Lorentz rotations.

$$
\rho(t) \psi_{\mathrm{p}}=\mathrm{m}(\mathrm{t}, \mathrm{p}) \psi_{\mathrm{p}}
$$


For each $p$, the map $t \rightarrow m(t, p)$ is a homomorphism of $T$ into the complex numbers (since $T$ acts on $M$ trivially, i.e., $T \subset G^{p}$ for all $\left.p \in M\right) m(t, p)$ is determined explicitly in the following way: The Lie algebra $\mathrm{T}$ of $\mathrm{T}$ can be identified with the space-time vector space, the space of momenta is its dual space. Then, $m(\operatorname{Exp} X, p)=e^{i p(X)}$ for $x \in T, p \in R^{4}$.

The spin representations of $G$ require a slightly more elaborate formalism, that will be described later when it is needed.

Let $\mathrm{H}$ continue to be a Hilbert space, $\mathrm{p} \rightarrow \psi_{\mathrm{p}}, \mathrm{p} \in \mathrm{M}$, an "orthonormal basis" for $H$ parameterized by the measure space $M$. Suppose that $M$ is also a topological space. We can now define the notion of the support of an element $\psi^{i} \in \mathrm{D}$.

Let $\mathrm{U}$ be an open subset of $\mathrm{M}$. One says that an element $\psi \epsilon \mathrm{H}$ vanishes outside $U$ if

$$
<\psi \mid \psi_{\mathrm{p}}>=0 \quad \text { for all } \mathrm{p} \in \mathrm{m}-\mathrm{U}
$$

Let $\psi$ be an element of $D$. Let us say that $\psi^{\prime}$ vanishes inside $U$ if:

$$
<\psi^{\prime}>=0 \text { for all } \psi \in \mathrm{H} \text { which vanish outside } \mathrm{U} \text {. }
$$

Say that an element $\psi^{r} \in \mathrm{D}$ vanishes in a neighborhood of a point $p \in M$ if there is an open subset containing $p$ in which $\psi^{\prime}$ vanishes. The union of all such points is then an open set, hence its complement in $M$ is a closed subset of $M$ that we will call the support of $\psi^{\prime}$.

Let us now apply these general notions to an example where everything is sufficiently simple and, in principle, known, yet still sufficiently illustrative to suggest further developments. 
VII. PARTIAL WAVE ANALYSIS FOR S-MATRICES ASSOCIATED WITH SPIN ZERO REPRESENTATIONS OF SEMIDIRECT PRODUCT GROUPS

We now want to examine the usual derivation of the partial wave analysis of the scattering operator in order to isolate certain interesting group-theoretic features.

Suppose that $G$ is a semidirect product of an abelian invariant subgroup $\mathrm{T}$ and a subgroup $\mathrm{L}$, i.e., $\mathrm{G}=\mathrm{L} \cdot \mathrm{T}$. (Of course, as our notations indicate, again we have in mind the case: $\mathrm{G}=$ Poincaré group, $\mathrm{L}=$ Lorentz subgroup, $\mathrm{T}=$ translation subgroup.) Use the notations described in the last section: $\mathrm{H}_{1}, \ldots, \mathrm{H}_{4}$ are Hilbert spaces, $\rho_{1}, \ldots, \rho_{4}$ unitary representations of $\mathrm{G}$ on $\mathrm{H}_{1}, \ldots, \mathrm{H}_{4}$ which extend to $\mathrm{D}_{1}, \ldots, \mathrm{D}_{4}$ Dirac spaces associated with $\mathrm{H}_{1}, \ldots, \mathrm{H}_{4}$. Suppose $\mathrm{M}_{1}, \ldots, \mathrm{M}_{4}$ are auxiliary measure spaces in which $\mathrm{L}$ acts with

$$
\mathrm{p}_{\mathrm{i}} \rightarrow \psi_{\mathrm{p}_{\mathrm{i}}} \quad \mathrm{i}=1, \ldots, 4
$$

defining orthonormal basis for $\mathrm{H}_{1}, \ldots, \mathrm{H}_{4}$ with

$$
\begin{aligned}
& \rho_{\mathrm{i}}(\mathrm{t})\left(\psi_{\mathrm{p}_{i}}\right)=\mathrm{m}_{\mathrm{i}}\left(t, \mathrm{p}_{\mathrm{i}}\right) \quad \psi_{\mathrm{p}_{\mathrm{i}}} \\
& \rho_{\mathrm{i}}(\ell)\left(\psi_{\mathrm{p}_{\mathrm{i}}}\right)=\psi_{\ell \mathrm{p}_{\mathrm{i}}} \\
& 1 \leq \mathrm{i} \leq 4, \quad \mathrm{t} \in \mathrm{T}, \quad \ell \in \mathrm{L}
\end{aligned}
$$

For fixed $p_{i}, t \rightarrow m_{i}\left(t, p_{i}\right)$ is a homomorphism of $T$ into the complex numbers. Suppose $L$ acts transetively on $M_{1}, \ldots, M_{4}$. Let $p_{1}^{0}, \ldots, p_{4}^{o}$ be fixed points of $M_{1}, \ldots, M_{4}$ (the "rest frame"), and $K_{1}, \ldots, K_{4}$ the isotropy subgroup 
of $L$ at $\mathrm{p}_{1}, \ldots, \mathrm{p}_{4}$. Thus

$$
\mathrm{M}_{\mathrm{i}}=\mathrm{L} / \mathrm{K}_{\mathrm{i}}, \quad \mathrm{i}=1, \ldots, 4
$$

Now, suppose $A$ is an operator $\mathrm{H}_{1} \otimes \mathrm{H}_{2} \rightarrow \mathrm{H}_{3} \otimes \mathrm{H}_{4}$ that intertwines the tensor-product action of $G$ on each of the Hilbert spaces. Suppose also that $A$ extends to a mapping of the corresponding Dirac spaces, i.e.,

$$
\mathrm{A}: \mathrm{D}_{1} \otimes \mathrm{D}_{2} \rightarrow \mathrm{D}_{3} \otimes \mathrm{D}_{4}
$$

The customary analysis now proceeds to regard the following formula as defining a "function" of the indicated variables, despite the fact that it is not considered legitimate to define the inner product of two elements of the Dirac spaces:

$$
\mathrm{f}\left(\mathrm{p}_{3}, \mathrm{p}_{4}\right)=<\mathrm{A}\left(\psi_{\mathrm{p}_{1}^{\circ}} \otimes \psi_{\mathrm{p}_{2}^{\circ}}\right) \mid \psi_{\mathrm{p}_{3}} \otimes \psi_{\mathrm{p}_{4}}>
$$

Then, for $t \in T$,

$$
\begin{aligned}
\mathrm{f}\left(\mathrm{p}_{3}, \mathrm{p}_{4}\right) & =\left\langle\left(\rho_{1} \otimes \rho_{2}\right)(t)\left(\mathrm{A}\left(\psi_{\mathrm{p}_{1}^{\circ}} \otimes \psi_{\mathrm{p}_{2}^{\circ}}\right)\right) \mid\left(\rho_{3} \otimes \rho_{4}\right)(t)\left(\psi_{\mathrm{p}_{3}} \otimes \psi_{\mathrm{p}_{4}}\right)\right\rangle \\
& =m_{1}\left(t, \mathrm{p}_{1}^{o}\right) * \mathrm{~m}_{2}\left(t, \mathrm{p}_{2}^{\circ}\right) * \mathrm{~m}_{3}\left(t, \mathrm{p}_{3}\right) \mathrm{m}_{4}\left(t, \mathrm{p}_{4}\right) \mathrm{f}\left(\mathrm{p}_{3}, \mathrm{p}_{4}\right)
\end{aligned}
$$

This identity seems to imply that:

$$
\begin{aligned}
& \mathrm{f}\left(\mathrm{p}_{3}, \mathrm{p}_{4}\right)=0 \text {, except when } \\
& \mathrm{m}_{3}\left(t, \mathrm{p}_{3}\right) \mathrm{m}_{4}\left(t, \mathrm{p}_{4}\right)=\mathrm{m}_{1}\left(t, \mathrm{p}_{1}^{\mathrm{o}}\right) \mathrm{m}_{2}\left(t, \mathrm{p}_{2}^{\circ}\right)
\end{aligned}
$$


Let $\mathrm{N}$ be the set of points $\left(\mathrm{p}_{3}, \mathrm{p}_{4}\right)$ of $\mathrm{M}_{3} \times \mathrm{M}_{4}$ satisfying condition (8.3). The correct interpretation of this condition should be then that the support of the Dirac element $\mathrm{A}\left(\psi_{\mathrm{p}_{1}^{\mathrm{o}}} \otimes \psi_{\mathrm{p}_{2}^{\circ}}\right)$ of $\mathrm{H}_{3} \otimes \mathrm{H}_{4}$ is contained in the set $\mathrm{N}$. Another condition is readily obtained: Let $K$ be the group $K_{1} \cap K_{2}$, i.e.,

$$
\dot{\rho(\mathrm{k})}\left(\psi_{\mathrm{p}_{1}^{\circ}} \otimes \psi_{\mathrm{p} g}\right)=\psi_{\mathrm{p}_{1}^{\circ}} \otimes \psi_{\mathrm{p}_{2}^{\circ}} \text { for } \mathrm{k} \epsilon \mathrm{K}
$$

Then, we have, from (8.2),

$$
\mathrm{f}\left(\mathrm{kp}_{3}, \mathrm{kp} \mathrm{p}_{4}\right)=\mathrm{f}\left(\mathrm{p}_{3}, \mathrm{p}_{4}\right)
$$

i.e., if $f\left(p_{3}, p_{4}\right)$ is a genuine function of the subset $N$ of $M_{1} \times M_{2}$, it is invariant under the action of $\mathrm{K}$.

Let us examine the condition (8.3) that determines $N$. Now, for fixed $p_{j} \in M_{i}, \quad(j=1,2,3,4), t \rightarrow m_{j}\left(t, p_{j}\right)$ determines a homomorphism of the translation group $\mathrm{T}$ into the complex numbers of absolute value 1 . Let $\mathrm{T}$ be the Lie algebra of $T$, and let $T^{d}$ be the dual space of $T$, i.e., the space of linear mappings of the vector space $T$ into the real numbers. Then, each $p_{i}$ determines an element of $T^{d}$ (for which we use the same notation) such that for $\mathrm{X} \in \mathrm{T}$ :

$$
m_{j}\left(\operatorname{Exp} X, p_{j}\right)=e^{i<p_{j}, X>}
$$

( $<\mathrm{p}_{\mathrm{j}}, \mathrm{X}>$ denotes the value the linear form $\mathrm{p}_{\mathrm{j}}$ takes on the element $\mathrm{X} \in \mathrm{T}_{\mathrm{.}}$ ) For $\ell \in \mathrm{L}$, Ad $\ell$ maps $\mathrm{T}$ into $\mathrm{T}$. Let $\mathrm{Ad}^{\mathrm{d}} \ell$ denote the dual representation in $\mathrm{T}^{\mathrm{d}}$, i.e.,

$$
<\operatorname{Ad}^{\mathrm{d}} \ell(\mathrm{p}), \mathrm{X}>=<\mathrm{p}, \mathrm{Adl}^{-1}(\mathrm{X})>\text { For } \ell \in \mathrm{L}, \mathrm{X} \in \mathrm{T}
$$


(8.3) takes the form:

$$
e^{i<p_{3}+p_{4}, X>}=e^{i<p_{1}^{o}+p_{2}^{o}, x>}, \text { i.e., }
$$

$\left(\mathrm{p}_{3}, \mathrm{p}_{4}\right) \in \mathrm{N}$ if and only if: $\mathrm{p}_{3}+\mathrm{p}_{4}=\mathrm{p}_{1}^{\mathrm{o}}+\mathrm{p}_{2}^{\mathrm{o}}$

In the physicist's notation, this takes the customary form for the "S-matrix":

$$
<p_{1}, p_{2}|A| p_{3}, p_{4}>=\delta\left(p_{1}+p_{2}-p_{3}-p_{4}\right) f(q) \text {, where } f(q) \text { is function }
$$

on the subset $\mathrm{N} \subset \mathrm{M}_{1} \times \mathrm{M}_{2} \times \mathrm{M}_{3} \times \mathrm{M}_{4}$ determined by (8.5). This "invariant amplitude" $f(q)$ is not an arbitrary function on $N$, but is invariant under the group $K\left(p_{1}, p_{2}\right)$, which is the intersection $K\left(p_{1}\right) \cap K\left(p_{2}\right)$, where $K\left(p_{j}\right)$ is the subgroup of the $\ell \in I$ such that $A d^{d} \ell\left(p_{j}\right)=p_{j}$.

The usual "partial wave expansion" for the invariant amplitudes should now be interpreted as an expansion for $f$ in terms of a complete, orthonormal basis of functions on $\mathrm{N}$ which are invariant under $\mathrm{K}\left(\mathrm{p}_{1}, \mathrm{p}_{2}\right)$. If $\mathrm{N}$ is compact, this should be expected to be an expansion over a discrete parameter, but if $\mathrm{N}$ is non-compact, it may be expected to be an expansion over a continuous parameter, similiar to the physicist's expansion of the amplitude after the Sommerfeld-Watson transform. The relevance of our remarks in Section 6 is a simple mathematical model for this phenomonon should now be clear.

In summary, we have gone through the analysis to point out the existence of two general mathematical problems, which we will now state, but will only work on seriously in later papers:

\section{Problem I}

Suppose $\rho$ is a unitary representation of a group $G$ on a Hilbert space $H$, which extends to a representation of $G$ on a suitable Dirac space $D$ associated 
with $\mathrm{H}$. Suppose $\mathrm{G}^{\prime}$ is a subgroup of $\mathrm{G}$, and $\psi \in \mathrm{D}$ satisfies:

$$
\rho(\mathrm{g})(\psi)=\mathrm{m}^{\prime}(\mathrm{g}) \psi^{\prime} \text { for } \mathrm{g} \in \mathrm{G}^{\prime} \quad \text {. }
$$

Suppose $\mathrm{p} \rightarrow \psi_{\mathrm{p}} \in \mathrm{D}, \mathrm{p} \in \mathrm{M}$, is an orthonormal basis of $\mathrm{D}$, satisfying:

$$
\rho(\mathrm{g}) \psi_{\mathrm{p}}=\mathrm{m}(\mathrm{g}, \mathrm{p}) \psi_{\mathrm{p}} \quad \text { for } \mathrm{g} \in \mathrm{G}, \mathrm{p} \in \mathrm{M}
$$

Write formally:

$$
\mathrm{f}(\mathrm{g})=\left\langle\psi^{\prime} \mid \psi_{\mathrm{p}}\right\rangle
$$

It appears that:

$$
f(g p)=m^{\prime}(g) m(g, p) f(p) \quad \text { for } g \in G^{\prime}, p \in M
$$

In particular, for $g \in G^{p} \cap G^{\prime}\left(G^{p}=\right.$ isotropy subgroup of $G$ at $p$ ),

$$
f(p)=m^{\prime}(g) m(g, p) f(p)
$$

Guided by our experience with the "S-matrix" case, we would guess that this identity requires that: The support of $\psi$ be in the set of all points $p \in \mathrm{M}$ such that

$$
m^{\prime}(g) m(g, p)=1 \quad \text { for } g \in G^{p} \cap G^{\prime}
$$

Apparently, this sort of problem has not at all been considered (at least at this level of generality) in the mathematical literature. However, there is a close relation to problems considered by $\mathrm{F}$. Bruhat [1]. 


\section{Problem II}

Let $M$ be a manifold, $N^{\lambda}$ a submanifold of $M$ depending smoothly on the parameter $\lambda_{0}$. (In the "S-matrix" case, $\lambda$ may be considered as the initial momenta $\left.\left(p_{1}^{0}, p_{2}^{o}\right)\right)$. Let $L$ be a group which acts on $M$. Suppose $K^{\lambda}$ is a subgroup $L$ (also depending smoothly on $\lambda$ ) such that $K^{\lambda}$ maps the subset $\mathrm{N}^{\lambda}$ into itself. Consider a function $\mathrm{f}^{\lambda}$ defined on $\mathrm{N}^{\lambda}$, invariant under $\mathrm{K}^{\lambda}$. The problem: Investigate the expansion of $\mathrm{f}^{\lambda}$ in terms of a complete, orthonormal basis for functions on $\mathrm{N}^{\lambda}$, particularly in regard to the dependence on $\lambda$. For example, the variation through values of $\lambda$ at which $\mathrm{N}^{\lambda}$ changes from a compact to a non-compact set should be particularly interesting. Recent work by D. Freedman and J. M. Wang [6], M. Goldberger and C. E. Jones [7], and E. Leader (to be published) should particularly be examined in this spirit. 


\section{ANALYTIC CONTINUATION OF MATRIX ELEMENTS OF $\operatorname{SO}(2,1)$}

As a guide to our general problem of investigation the asymptotic behavior

of matrix elements of the form $\left\langle\psi^{\prime}\right| U(t) \psi>$ as $t \rightarrow \infty$, we examine in more detail in this section the case where $U(t)$ is a one-parameter group generated by one of the elements of the Lie algebra of $\operatorname{SO}(2,1)$. This will be done in such a way as to lead into certain general features to be covered in Sections XI and XII.

Again we will work with the "Gell-Mann formula" method of generating representations of $\mathrm{SL}(2, \mathrm{R})$, which we will formulate in a slightly different manner.

Let $\mathrm{H}$ be a Hilbert space, with $\mathrm{Z}, \mathrm{X}, \mathrm{Y}$ operators on $\mathrm{H}$ such that:

$$
[\mathrm{Z}, \mathrm{X}]=\mathrm{Y} ;[\mathrm{Z}, \mathrm{Y}]=-\mathrm{X}[\mathrm{X}, \mathrm{Y}]=0 ; \mathrm{X}^{2}+\mathrm{Y}^{2}=-1
$$

Define operators $\mathrm{X}_{\lambda}=\mathrm{YZ}+\lambda \mathrm{X}$

$$
\mathrm{Y}_{\lambda}=\mathrm{XZ}+\lambda \mathrm{Y}
$$

Our goal is to calculate matrix elements of the form:

$$
f(t, \lambda)=\left\langle\psi^{\prime} \mid \operatorname{Exp}\left(t x_{\lambda}\right) \psi\right\rangle
$$

in such a form that the asymptotic behavior as $t \rightarrow \infty$ can be investigated.

Now, $\operatorname{Exp}\left(\operatorname{tx}_{\lambda}\right)$ satisfies the differential equation:

$$
\begin{aligned}
\frac{d}{d t} \operatorname{Exp}\left(\mathrm{tX}_{\lambda}\right) & =\mathrm{X}_{\lambda} \operatorname{Exp}\left(\mathrm{tX}_{\lambda}\right) \\
& =\left(\mathrm{X}_{0}+\lambda \mathrm{X}\right) \operatorname{Exp}\left(t \mathrm{X}_{\lambda}\right)
\end{aligned}
$$

Theorem 9.1

$$
\text { Set } \begin{aligned}
A_{\lambda}(t) & =Y^{\lambda} \operatorname{Exp}\left(t X_{0}\right) Y^{-\lambda} \ldots \text { Then } \\
\frac{d A_{\lambda}}{d t} & =\left(X_{0}+\lambda X\right) A_{\lambda}, A(0)=1
\end{aligned}
$$


In particular, $A_{\lambda}(t)$ satisfies the same differential equation (9.1) as $\operatorname{Exp}\left(t, x_{\lambda}\right)$, hence, if the relevant uniqueness theorem holds, then

Proof:

$$
\begin{aligned}
& \operatorname{Exp}\left(\mathrm{tX}_{\lambda}\right)=\mathrm{Y}^{\lambda} \operatorname{Exp}\left(\mathrm{tX}_{\mathrm{O}}\right) \mathrm{Y}^{-\lambda} \\
& {\left[\mathrm{X}_{\mathrm{O}}, \mathrm{Y}\right]=[\mathrm{YZ}, \mathrm{Y}]=-\mathrm{YX}}
\end{aligned}
$$

Since YX commutes with $\mathrm{Y}$, we have:

$$
\left[X_{0}, Y^{\lambda}\right]=\lambda Y^{\lambda-1}\left[X_{0}, Y\right]=-\lambda Y^{\lambda} X
$$

Hence,

$$
\begin{aligned}
& X_{0} Y^{\lambda} \operatorname{Exp}\left(t X_{0}\right) Y^{-\lambda}=\left[X_{0}, Y^{\lambda}\right] \operatorname{Exp}\left(t X_{0}\right) Y^{-\lambda} \\
& +Y^{\lambda} X_{0} \operatorname{Exp}\left(t X_{0}\right) Y^{-\lambda}=-\lambda Y^{\lambda} X \operatorname{Exp}\left(t X_{o}\right) Y^{-\lambda} \\
& +Y^{\lambda} X_{0} \operatorname{Exp}\left(t X_{0}\right) Y^{-\lambda}, \text { or }\left(X_{0}+\lambda X\right)\left(Y^{\lambda} \operatorname{Exp}\left(t X_{o}\right) Y^{-\lambda}\right) \\
& =Y^{\lambda} X_{o} \operatorname{Exp}\left(t X_{o}\right) Y^{-\lambda}=\frac{d A_{\lambda}}{d t}
\end{aligned}
$$

This proves (9.2), and the theorem.

In the next two sections, we will investigate the roots of this formula in Lie group cohomology. Let us now proceed to see how it helps to calculate matrix elements.

Let us now realize this algebra as an algebra of differential operators, using the method described in [12], Section VI. H is the Hilbert space of functions $\psi(\theta), 0 \leq \theta \leq 2 \pi$, with $\langle\psi \mid \psi\rangle=\int_{0}^{2 \pi} \psi(\theta)^{*} \psi(\theta) \mathrm{d} \theta$

$$
\begin{aligned}
& \mathrm{Z}=\frac{\mathrm{d}}{\mathrm{d} \theta} \\
& \mathrm{X}^{\lambda}=\sin \theta \frac{\mathrm{d}}{\mathrm{d} \theta}-\lambda \cos \theta \\
& \mathrm{Y}^{\lambda}=\cos \theta \frac{\mathrm{d}}{\mathrm{d} \theta}+\lambda \sin \theta
\end{aligned}
$$


Hence, $\mathrm{X}_{\mathrm{O}}=\sin \theta \frac{\mathrm{d}}{\mathrm{d} \theta}, \mathrm{Y}_{\mathrm{O}}=\sin \theta$

It is convenient to change variables:

$$
\begin{gathered}
z=e^{i \theta} \quad X_{0}=1 / 2\left(z^{2}-1\right) \frac{d}{d z}, Y=1 / 2 i\left(z-z^{-1}\right) \\
\operatorname{Exp}\left(t X_{0}\right)(z)=\left(z\left(\frac{1+e^{t}}{e^{t}-1}\right)+1\right) /\left(z+\left(\frac{1+e^{t}}{e^{t}-1}\right)\right)=\frac{z S+1}{z+s}, \text { with } s=\frac{1+e^{t}}{e^{t}-1}
\end{gathered}
$$

Note that $\operatorname{Exp}\left(t X_{0}\right)(z) \rightarrow \pm 1$ as $t \rightarrow \infty, s \rightarrow \pm 1$.

Note also that $s$ is the "correct" function of $t$ to choose to make

$\operatorname{Exp}\left(t(s) X_{0}(z)\right)$ analytic in the neighborhood of $t= \pm \infty$, i.e., $s= \pm 1$.

Suppose $\psi=z^{\mathrm{n}}$

$$
\begin{aligned}
& \operatorname{Exp}\left(\operatorname{tx} \mathrm{O}_{0}\right)\left(\mathrm{Y}^{-\lambda} \psi\right) \\
& =\operatorname{Exp}\left(\operatorname{tX} \mathrm{o}_{0}\right)\left(1 / 2 \mathrm{i}\left(\mathrm{z}-\mathrm{z}^{-1}\right)\right)^{-\lambda} \mathrm{z}^{\mathrm{n}}
\end{aligned}
$$

Now,

$$
\begin{aligned}
& \operatorname{Exp}\left(t X_{o}\right)\left(z-z^{-1}\right)=\operatorname{Exp}\left(t X_{o}\right)\left(\frac{z^{2}-1}{z}\right) \\
& =\frac{\left(\frac{z s+1}{z+s}\right)^{2}-1}{\frac{z s+1}{(z+s)}}=\frac{(z s+1)^{2}-(z+s)^{2}}{(z+s)(z s+1)} \\
& =\frac{z^{2} s^{2}+1+2 z s-z^{2}-2 s z-s^{2}}{z^{2} s+z s^{2}+z+s}=\frac{z^{2}\left(s^{2}-1\right)-\left(s^{2}-1\right)}{z^{2} s+z\left(s^{2}+1\right)+s}=\frac{\left(s^{2}-1\right)\left(z^{2}-1\right)}{z^{2} s+z\left(s^{2}+1\right)+s}
\end{aligned}
$$

Note that:

$$
\frac{\operatorname{Exp}\left(t X_{o}\right) Y \operatorname{Exp}\left(-t X_{0}\right)}{\left(s^{2}-1\right)} \text { is analytic at } s= \pm 1
$$


Hence,

$$
\begin{gathered}
\mathrm{Y}^{\lambda} \operatorname{Exp}\left(\mathrm{tX}_{\mathrm{o}}\right)\left(\mathrm{Y}^{-\lambda} \psi\right) \\
=\left(\frac{\mathrm{z}^{2}-1}{\mathrm{z}}\right)^{\lambda} \frac{\left(\mathrm{s}^{2}-1\right)^{-\lambda}\left(\mathrm{z}^{2}-1\right)^{-\lambda}}{\mathrm{z}^{2} \mathrm{~s}+\mathrm{z}\left(\mathrm{s}^{2}+1\right)+\mathrm{s}}\left(\frac{\mathrm{sz}+1}{\mathrm{z}+\mathrm{s}}\right)^{\mathrm{n}} \\
=\left(\mathrm{s}^{2}-1\right)^{-\lambda}\left(\frac{\mathrm{z}}{\mathrm{z}^{2} \mathrm{~s}+\mathrm{z}\left(\mathrm{s}^{2}+1\right)+\mathrm{s}}\right)^{-\lambda}\left(\frac{\mathrm{sz}+1}{\mathrm{z}+\mathrm{s}}\right)^{\mathrm{n}}
\end{gathered}
$$

Thus, we have proved:

Theorem 9.2 Suppose $\psi_{\mathrm{n}}, \psi_{\mathrm{m}}$ satisfy:

$$
\mathrm{Z}\left(\psi_{\mathrm{n}}\right)=\text { in } \psi_{\mathrm{n}}, \mathrm{Z}\left(\psi_{\mathrm{m}}\right)=\mathrm{im} \psi_{\mathrm{m}}
$$

Then, the matrix element:

$$
\left\langle\psi_{\mathrm{m}} \mid\left(\operatorname{Exp}\left(\mathrm{tx}_{\lambda}\right)\right) \psi_{\mathrm{n}}\right\rangle=\mathrm{f}(\mathrm{t}, \lambda)
$$

admits the following integral representation:

$$
\begin{aligned}
& f\left(\log \left(\frac{1+s}{1-s}\right), \lambda\right)=\left\langle\psi_{m} \mid Y^{\lambda} \operatorname{Exp}\left(t X_{o}\right) Y^{-\lambda} \psi_{n}\right\rangle \\
& =\frac{\left(s^{2}-1\right)^{-\lambda}}{i} \int\left(\frac{z}{z^{2} s+z\left(s^{2}+1\right)+s}\right)^{-\lambda}\left(\frac{s z+1}{z+s}\right)^{n} z^{-m+1} d z
\end{aligned}
$$


where the integral is taken over the unit circle in the comples z-plane. (Of course, since the integrand is analytic, it can be shifted in accordance with Cauchy's theorem.) Note particularly that $\left(s^{2}-1\right)^{\lambda} \mathrm{f}\left(\log \left(\frac{1+r}{1-s}\right), \lambda\right)$ has no singularity in the neighborhood of $s= \pm 1$.

This theorem sums up many special facts about the Legendre functions and their asymptotic behavior as $t \rightarrow \infty$. Another interesting observation: For certain values of complex $t$, there will be trouble with singularities in the integrand. This indicates the feature we pointed out earlier, $\operatorname{Exp}\left(\operatorname{tX}_{\lambda}\right)$ will not be strictly definable for complex $t$. 


\section{LIE GROUP COHOMOLOGY}

\section{AND THE DEFORMIATION OF REPRESENTATIONS}

Many features of the argument of the last section are of general interest. Since the argument implicitly involves Lie group cohomology, we will detour to sketch what we need.

Let $\mathrm{G}$ be a group, and let $\phi$ be a representation by linear transformations on a vector space $\mathrm{V}$. One will find an exposition of the associated cohomology groups and their algebraic interpretation in [14], Chapter 12. Since we will only need cohomology of degrees one and two, and our notation differs from those of Kurosh, we will sketch what we need. Work by van Est [4] indicates the relation between Lie group and Lie algebra cohomology.

A 0 -cochain is an element of $V$. A one-cochain is a map $\omega$ from $G$ to $V$, a two-cochain will be a map from $G \times G$ to $V$. These form vector spaces, that we denote by $C^{0}(\phi), C^{1}(\phi)$ and $C^{2}(\phi)$. A coboundary operator will send $C^{n}(\phi)$ into $\mathrm{C}^{\mathrm{n}+1}(\phi)$ :

(a) For $\omega \in \mathrm{C}^{\mathrm{O}}(\phi), \mathrm{i}, \mathrm{e}_{\circ}, \omega \in \mathrm{V}$,

$$
\mathrm{d} \omega(\mathrm{g})=\phi(\mathrm{g})(\omega)^{-\omega} \text { for } \mathrm{g} \in \mathrm{G}
$$

(b) For $\omega \in \mathrm{C}^{1}(\phi)$

$$
\mathrm{d} \omega\left(\mathrm{g}_{1}, \mathrm{~g}_{2}\right)=\omega\left(\mathrm{g}_{1}\right)+\phi\left(\mathrm{g}_{1}\right)\left(\omega\left(\mathrm{g}_{2}\right)\right)-\omega\left(\mathrm{g}_{1} \mathrm{~g}_{2}\right) \text { for } \mathrm{g}_{1}, \mathrm{~g}_{2} \in \mathrm{G}
$$

Let us show that $d(d \omega)=0$ for $\omega \in \mathrm{C}^{\circ}(\phi)$

$$
\begin{gathered}
\mathrm{d}(\mathrm{d} \omega)\left(\mathrm{g}_{1}, \mathrm{~g}_{2}\right)=\mathrm{d} \omega\left(\mathrm{g}_{1}\right)+\phi\left(\mathrm{g}_{1}\right)\left(\mathrm{d} \omega\left(\mathrm{g}_{2}\right)\right)-\mathrm{d} \omega\left(\mathrm{g}_{1}, \mathrm{~g}_{2}\right) \\
=\omega+\phi\left(\mathrm{g}_{1}\right)(\omega)+\phi\left(\mathrm{g}_{1}\right)\left(-\omega+\phi\left(\mathrm{g}_{2}\right)(\omega)\right)-\left(-\omega+\phi\left(\mathrm{g}_{1}, \mathrm{~g}_{2}\right)(\omega)\right)=0
\end{gathered}
$$

Let $\mathrm{Z}^{1}(\phi)$ be the kernel of $\mathrm{d}: \mathrm{C}^{1}(\phi) \rightarrow \mathrm{C}^{2}(\phi)$

Then $H^{1}(\phi)=\mathrm{Z}^{2}(\phi) / \mathrm{dC}^{\circ}(\phi)$. 
If $G$ is a Lie group and we restrict ourselves to cochains that are differentiable functions on $G$, then the connection with Lie algebra cohomology can be made explicit. Let $\mathrm{G}$ be the Lie algebra of $\mathrm{G}$, and let $\mathrm{X} \in \mathrm{G}$. The representation $\phi$ of $G$ induces a Lie algebra representation by operators on $V$, that we will denote by $\phi$, of $\mathrm{G}$ :

$$
\phi(x)=\frac{d}{d t} \phi(\operatorname{Exp}(t X)) / t=0
$$

We can define mapping $\alpha: C^{r}(\phi) \rightarrow C^{r}(\phi), r=0,1,1$, that commute the d-operator.

$$
\mathrm{C}^{\mathrm{O}}(\phi) \text { and } \mathrm{C}^{\mathrm{O}}(\phi) \text { are both equal to } \mathrm{V} \text {, hence identified. }
$$

If $\omega \in \mathrm{C}^{1}(\phi)$, define $\alpha(\omega)$ as follows:

$$
d \alpha(\omega)(X)=\frac{d}{d t} \omega(\operatorname{Exp}(\mathrm{tX})) /_{t=0}
$$

Suppose that $\omega \in V$

$$
\begin{aligned}
\mathrm{d} \omega(\mathrm{g}) & =-\omega+\phi(\mathrm{G})(\omega), \text { hence } \alpha(\mathrm{d} \omega)(\mathrm{X})=\frac{\mathrm{d}}{\mathrm{dt}}(\omega+\phi(\operatorname{Exp}(\mathrm{tX}))(\omega)) /_{\mathrm{t}=0} \\
& =\phi(\mathrm{X})(\omega)=\mathrm{d} \omega(\mathrm{X}),
\end{aligned}
$$

where $d \omega$ is taken in the sense of Lie algebra cohomology. Then

$$
\alpha \mathrm{d} \omega=\mathrm{d} \alpha \omega \text { for } \omega \in \mathrm{C}^{\circ}(\phi)
$$

Let us consider $\omega \in \mathrm{C}^{1}(\phi)$

$$
\begin{gathered}
\mathrm{d} \alpha(\omega)(\mathrm{X}, \mathrm{Y})=\phi(\mathrm{X})(\alpha(\omega)(\mathrm{Y}) \alpha(\omega)(\mathrm{X}))-\alpha(\omega)([\mathrm{X}, \mathrm{Y}]) \\
=\frac{\mathrm{d}}{\mathrm{dt}} \phi(\mathrm{X}) \omega(\operatorname{Exp}(\mathrm{tY}))-\phi(\mathrm{Y}) \omega(\operatorname{Exp}(\mathrm{tX}))-\omega(\operatorname{Exp}(\mathrm{t}[\mathrm{X}, \mathrm{Y}])) /_{\mathrm{t}=0} \\
=\frac{\partial}{\partial t} \frac{\partial}{\partial \mathrm{s}} \phi(\operatorname{Exp}(\mathrm{sX})) \omega(\operatorname{Exp}(\mathrm{tY}))-\phi(\operatorname{Exp}(\mathrm{SY})) \omega(\operatorname{Exp}(\mathrm{tX})) / \mathrm{s}, \mathrm{t}=0 \\
-\frac{\mathrm{d}}{\mathrm{dt}} \omega(\operatorname{Exp}(\mathrm{t}[\mathrm{X}, \mathrm{Y}])) / \mathrm{t}=0 \\
=\frac{\partial}{\partial t} \frac{\partial}{\partial s} \mathrm{~d} \omega(\operatorname{Exp}(\mathrm{SX}), \operatorname{Exp}(\mathrm{tY}))-\omega(\operatorname{Exp}(\mathrm{tY}))+\omega(\operatorname{Exp}(\mathrm{sX}), \operatorname{Exp}(\mathrm{tY}))-\mathrm{d} \omega \\
(\operatorname{Exp}(\mathrm{sY}), \operatorname{Exp}(\mathrm{tX}))+\omega(\operatorname{Exp}(\mathrm{tX}))-\omega(\operatorname{Exp}(\mathrm{SY}), \operatorname{Exp}(\mathrm{tX})) / \mathrm{s}, \mathrm{t}=0
\end{gathered}
$$




$$
-\frac{\mathrm{d}}{\mathrm{dt}} \omega(\operatorname{Exp}(\mathrm{t}[\mathrm{X}, \mathrm{Y}])) / \mathrm{t}=0
$$

We now prove that:

$$
\begin{aligned}
& \frac{\partial}{\partial t} \frac{\partial}{\partial s}[\omega(\operatorname{Exp}(\operatorname{SX}) \operatorname{Exp}(t Y))-\omega(\operatorname{Exp}(S Y) \operatorname{Exp}(\mathrm{XX}))] r, t=0 \\
& \left.=\frac{\mathrm{d}}{\mathrm{d} t} \omega(\operatorname{Exp}(t[X, Y]))\right)_{t=0}
\end{aligned}
$$

For the proof, let us suppose that $G$ is a group of $n \times n$ matrices, and that $\omega$ can be extended to a function on all $\mathrm{n} \times \mathrm{n}$ matrices. (Using the techniques of manifold theory, the proof can be extended to abstractly given Lie groups.) The left-hand side of 10.1 is then:

$$
\frac{d}{d t}[\omega(X \operatorname{Exp}(\mathrm{tY}))-\omega(\mathrm{Y} \operatorname{Exp}(\mathrm{tX}))] / \mathrm{t}=0
$$

Consider the following curve in the space of $n \times n$ matrices:

$$
\begin{aligned}
& x(t)=X \operatorname{Exp}(t Y) \\
& y(t)=Y \operatorname{Exp}(t X) \\
& z(t)=\operatorname{Exp}(t[X, Y])
\end{aligned}
$$

Then,

$$
x^{\prime}(0)=X Y ; y^{\prime}(0)=Y X ; z^{\prime}(0)=[X, Y] \text {, i. e. }, X^{\prime}(0)-y^{\prime}(0)=z^{\prime}(0)
$$

This relation between the tangent vectors of the three curves leads to the relation among their directional derivatives.

$$
\frac{d}{d t} \omega(x(t))-\frac{d}{d t} \omega(y(t))-\frac{d}{d t} \omega(z(t)) / t=0=0 \text {, which is just } 10.1 .
$$

10.1 enables us to see how to define $\alpha(\omega)$ if $\omega \in \mathrm{C}^{2}(\phi)$, namely:

$$
\begin{aligned}
& \alpha(\omega)(X, Y)=\frac{\partial^{2}}{\partial s \partial t} \omega(\operatorname{Exp}(\mathrm{SX}) \operatorname{Exp}(\mathrm{tY}))-\omega(\operatorname{Exp}(\mathrm{SY}) \operatorname{Exp}(\mathrm{tX})) / \mathrm{s}, \mathrm{t}=0 \\
& \text { for } \mathrm{X}, \mathrm{Y} \in \mathrm{G} \text {. }
\end{aligned}
$$


One sees from this formula that $\alpha(\omega)(\mathrm{X}, \mathrm{Y})=-\alpha(\omega)(\mathrm{Y}, \mathrm{X})$, i. e., $\alpha(\omega)$ is actually a cochain in $\mathrm{C}^{2}(\phi)$. Further, our method of defining $\alpha\left(\mathrm{C}^{2}(\phi)\right)$ guarantees that:

$$
\alpha(\mathrm{d} \omega)=\mathrm{d} \alpha(\omega) \text { for } \omega \in \mathrm{C}^{1}(\phi)
$$

Having shown the connection between Lie group and Lie algebra cohomology, let us turn to the connection between the former idea and deformation of Lie group representations.

Suppose $\mathrm{G}$ is a Lie group $\mathrm{g} \rightarrow \rho(\mathrm{g})$ is a representation of $\mathrm{G}$ by linear transformations on a vector space $H$. Consider a deformation of $\rho$ depending on a real parameter $\lambda$, i.e., for each $\lambda, g \rightarrow \rho_{\lambda}$ (g) is a representation of $G$ by linear transformations on $H$, reducing to the given one for $\lambda=0$.

$$
\text { Set } \omega(\mathrm{g})=\frac{\mathrm{d}}{\mathrm{d} \lambda} \rho_{\lambda}(\mathrm{g}) \rho\left(\mathrm{g}^{-1}\right) / \lambda=0
$$

Then,

$$
\begin{aligned}
\omega\left(\mathrm{g}_{1} \mathrm{~g}_{2}\right) & =\frac{\mathrm{d}}{\mathrm{d} \lambda}\left(\mathrm{P}_{\lambda}\left(\mathrm{g}_{1}\right) \rho_{\lambda}\left(\mathrm{g}_{2}\right)\right) \rho\left(\mathrm{g}_{2}^{-1}\right) \rho\left(\mathrm{g}_{1}^{-1}\right) /{ }_{\lambda=0} \\
& =\omega\left(\mathrm{g}_{1}\right)+\rho\left(\mathrm{g}_{1}\right) \omega\left(\mathrm{g}_{2}\right) \rho\left(\mathrm{g}_{1}^{-1}\right)
\end{aligned}
$$

Let $\mathrm{V}$ be the vector space of linear transformations: $\mathrm{H} \rightarrow \mathrm{H}$. Let $\phi$ be the following representation of $\mathrm{G}$ by linear transformations on $\mathrm{V}$ :

$$
\phi(g)(A)=\rho(g) A \rho\left(g^{-1}\right) \text { for } g \in G, A \in V .
$$

Regard $\omega$ as an element of $\mathrm{C}^{1}(\phi)$. Then, condition 10.2 means:

$$
\mathrm{d} \omega=0
$$

Define: $\omega(X)=\frac{d}{d \lambda} \phi_{\lambda}(X){ }_{\lambda}=0$ for $X \in \underset{N i}{G}$

Regard $\omega$ as an element of $\mathrm{C}^{1}(\phi)$.

Then, it is readily verified that: $\alpha(\omega)=\omega$,

i.e., the definition of $\omega$ as a cohomological "first obstruction" associated with 
the deformation $\lambda \rightarrow \rho_{\lambda}$ of Lie group representations is compatible with the previous definition (in [12], Part 2) of $\underset{\mathrm{m}_{\mathrm{m}}}{\omega}$ as a "first destruction" associated with the deformation $\lambda \longrightarrow \rho_{\lambda}$ of Lie algebra representations.

We now turn from this brief general review of material covered in detail by Nijenhuis and Richardson to a more specific topic, namely, the formulation in cohomological language of the argument used in Section 9. 
XI. GROUP REPRESENTATIONS DEFORMATIONS ASSOCIATED WITH THE LIE ALGEBRA REPRESENTATIONS THAT ARE LINEAR IN THE PARAMETER

Suppose $G$ is a connected Lie group, $\underset{m}{G}$ is its Lie algebra, and $\rho_{\lambda}$ is a one-parameter family of representations of $\mathrm{G}$ by linear transformations on a vector space H. Let $\underset{m}{\rho}=\rho_{0}$, and assume that $\rho_{\lambda}{ }_{\lambda}$ is of the form:

$$
\underset{m}{\rho} \lambda(\mathrm{X})=\rho(\mathrm{X})+\lambda \omega(\mathrm{X}) \quad \text { for } \mathrm{X} \in \underset{m}{\mathrm{G}}
$$

where $\omega \in \mathrm{C}^{1}(\phi) .(\mathrm{V}$ is the space of linear operators: $\mathrm{H} \rightarrow \mathrm{H}$, and $\phi(\mathrm{X})(\mathrm{A})=$ $\rho(X)-A \rho(X)=[\rho(X), A]$ for $X \in G, A \in V)$. Now, we know the conditions that $\stackrel{\rho}{m}$ given in this form be a representation, namely:

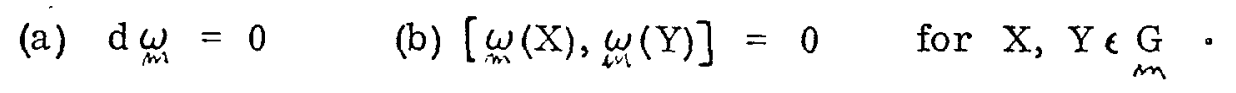

Our problem is to show that this special assumption about the Lie algebra deformation implies a special form of the group deformation, namely:

$$
\rho_{\lambda}(\mathrm{g})=\mathrm{A}(\mathrm{g}, \lambda) \rho(\mathrm{g})
$$

where, for each $\mathrm{g}, \lambda, \mathrm{A}(\mathrm{g}, \lambda)$ is a linear operator: $\mathrm{H} \rightarrow \mathrm{H}$, and $A(e, \lambda)=A(g, 0)=1$ (the identity operator).

First, let us start off with a deformation $\rho_{\lambda}(\mathrm{g})$ of form 11.3 and deduce the consequences.

$$
\begin{gathered}
\omega(\mathrm{g})=\frac{\mathrm{d}}{\mathrm{d} \lambda} \rho_{\lambda}(\mathrm{g}) \rho\left(\mathrm{g}^{-1}\right) / \lambda=0=\frac{\mathrm{d}}{\mathrm{d} \lambda} \mathrm{A}(\mathrm{g}, \lambda) /_{\lambda=0} \\
\rho_{\lambda}(\mathrm{X})=\frac{\mathrm{d}}{\mathrm{dt}} \rho_{\lambda}(\operatorname{Exp}(\mathrm{tX})) /_{\mathrm{t}=0}=\frac{\mathrm{d}}{\mathrm{dt}} \mathrm{A}(\operatorname{Exp}(\mathrm{tX}), \lambda) \rho(\operatorname{Exp}(\mathrm{tX})) /_{\mathrm{t}=0} \\
=\underset{m}{\mathrm{~A}}(\mathrm{X}, \lambda)+\rho_{m}(\mathrm{X}),
\end{gathered}
$$


with

$$
A(X, \lambda)=\frac{d}{d t} A(\operatorname{Exp}(t X), \lambda) /_{t=0} .
$$

Hence,

$$
A(X, \lambda)=\lambda \omega(X) \text { for } X \in G \text {, all } \lambda \text {. }
$$

Let us write down the condition that 11.3 define a representation for every value of $\lambda$ :

$$
\begin{aligned}
\rho_{\lambda}\left(\mathrm{g}_{1} \mathrm{~g}_{2}\right) & =\mathrm{A}\left(\mathrm{g}_{1} \mathrm{~g}_{2}, \lambda\right) \rho\left(\mathrm{g}_{1} \mathrm{~g}_{2}\right)=\rho_{\lambda}\left(\mathrm{g}_{1}\right) \rho_{\lambda}\left(\mathrm{g}_{2}\right) \\
& =\mathrm{A}\left(\mathrm{g}_{1}, \lambda\right) \rho\left(\mathrm{g}_{1}\right) \mathrm{A}\left(\mathrm{g}_{2}, \lambda\right) \rho\left(\mathrm{g}_{2}\right) \\
& =\mathrm{A}\left(\mathrm{g}_{1}, \lambda\right) \rho\left(\mathrm{g}_{1}\right) \mathrm{A}\left(\mathrm{g}_{2}, \lambda\right) \rho\left(\mathrm{g}_{2}^{-1}\right) \rho\left(\mathrm{g}_{1} \mathrm{~g}_{2}\right)
\end{aligned}
$$

or

$$
A\left(g_{1} g_{2}, \lambda\right)=A\left(g_{1}, \lambda\right) \rho\left(g_{1}\right) A\left(g_{2}, \lambda\right) \rho\left(g_{1}^{-1}\right) .
$$

Our experience with constructing "multipliers" for representations now suggests a way to solve these equations: As an Ansatz, set:

$$
A(g, \lambda)=\operatorname{Exp}(\lambda \omega(g))
$$

where:

$\omega \in \mathrm{C}^{1}(\phi)$, i. e., $\omega$ is a mapping of $\mathrm{G} \rightarrow \mathrm{V}=$ (operators on $\mathrm{H}$ )

$\{\omega(\mathrm{g}): \mathrm{g} \epsilon \mathrm{G}\}$ forms an abeljan set of operators on $\mathrm{H}$.

Then 11.7 takes the form:

$$
\begin{aligned}
\operatorname{Exp}\left(\lambda \omega\left(\mathrm{g}_{1} \mathrm{~g}_{2}\right)\right) & =\operatorname{Exp}\left(\lambda \omega\left(\mathrm{g}_{1}\right)\right) \rho\left(\mathrm{g}_{1}\right) \operatorname{Exp}\left(\lambda \omega\left(\mathrm{g}_{2}\right)\right) \rho\left(\mathrm{g}_{1}^{-1}\right) \\
& =\operatorname{Exp}\left(\lambda \omega\left(\mathrm{g}_{1}\right)+\rho\left(\mathrm{g}_{1}\right) \omega\left(\mathrm{g}_{2}\right) \rho\left(\mathrm{g}_{1}^{-1}\right)\right) .
\end{aligned}
$$


This condition is implied by the following one:

$$
\omega\left(g_{1} g_{2}\right)=\omega\left(g_{1}\right)+\phi\left(g_{1}\right)\left(\omega\left(g_{2}\right)\right)
$$

with $\rho \phi\left(\mathrm{g}_{1}\right)(\mathrm{A})=\rho\left(\mathrm{g}_{1}\right) \mathrm{A} \rho\left(\mathrm{g}_{1}^{-1}\right)$, for each $\mathrm{A} \in \mathrm{V}$, i. e., each linear

map A: $\mathrm{H} \rightarrow \mathrm{H} .11 .10$ is just the condition that $\mathrm{d} \omega=0$, where $\omega$ is interpreted as an element of $\mathrm{C}^{1}(\phi)$.

Then, 11.9 and 11.10 are sufficient conditions that 11.3 and 11.7 define a deformation $\lambda \rightarrow \rho_{\lambda}$ of representations of $\mathrm{G}$.

Suppose further that:

$$
\alpha(\omega)=d B, \text { for } B \epsilon V \text {, i. e., } B \epsilon C^{\circ}(\phi) \text {. }
$$

Then, we know that this is so if and only if :

$$
\omega=d B \text {, where } B \text { is interpreted as an element of } C^{\circ}(\phi) \text {. }
$$

Then,

$$
\begin{aligned}
\omega(\mathrm{g}) & =\mathrm{B}-\phi(\mathrm{g})(\mathrm{B}) \\
& =\mathrm{B}-\rho(\mathrm{g}) \mathrm{B} \rho\left(\mathrm{g}^{-1}\right)
\end{aligned}
$$

Summing up, we have proved:

Theorem 11.1

Suppose $\rho$ is a representation of the Lie group $G$ by linear transformations on $H$. Let $B$ be a linear transformation: $H \rightarrow H$, and define $\omega(\mathrm{g})$, for $\mathrm{g} \in \mathrm{G}$, by 11.11. Suppose that 11.9 is satisfied. Then the following formula defines a deformation $\lambda \rightarrow \rho_{\lambda}$ of $\rho$.

$$
\rho_{\lambda}(\mathrm{g})=\operatorname{Exp}\left(\lambda\left(-\mathrm{B}+\rho(\mathrm{g}) \mathrm{B} \rho\left(\mathrm{g}^{-1}\right)\right) \rho(\mathrm{g})\right.
$$


The corresponding deformation $\rho^{\lambda}$ of the Lie algebra representation is given by:

$$
\rho_{\lambda}(\mathrm{X})=\rho(\mathrm{X})+\lambda \omega(\mathrm{X})
$$

with

$$
\boldsymbol{\omega}(\mathrm{X})=\mathrm{dB}(\mathrm{X})=[\rho(\mathrm{X}), \mathrm{B}]
$$

This is the abstract version of the construction done in Section 10 in order to calculate the asymptotic behavior of the Legendre function $\mathrm{P}_{\ell}^{\mathrm{j}}$ (cos ht) as $t \rightarrow \infty$. We will now turn to the abstract version of the asymptotic formula itself.

\section{AN ABSTRACT VERSION OF THE ASYMPTOTIC FORMULA}

Suppose $H$ is a Hilbert space, with $\rho^{\lambda}$ a one-parameter family of representations of $\mathrm{G}$ by operators on $\mathrm{H}$ of form 11.12 .

For fixed elements $\mathrm{X} \in \mathrm{G}, \psi, \psi^{\dagger} \epsilon \mathrm{H}$, consider the matrix element:

$$
f(t, \lambda)=\left\langle\psi^{\prime}\right| \rho_{\lambda} \operatorname{Exp}(t X)(\psi)>
$$

Our experience with the calculation in Section 10 suggests simplifying 11.2 with a further Ansatz. Suppose that:

(a) The operators $\left\{\mathrm{B}, \rho(\mathrm{g}) \mathrm{B} \rho\left(\mathrm{g}^{-1}\right): \mathrm{g} \epsilon \mathrm{G}\right\}$ form an abelian set of operators.

(b) There is an operator C with:

$$
B=\log C \text {, i.e, } C=\operatorname{Exp} B
$$

Under the condition,

$$
\rho_{\lambda}(\mathrm{g})=\mathrm{C}^{-\lambda} \rho(\rho \mathrm{g}) \mathrm{C}^{\lambda}
$$

Then for $\psi \in \mathrm{H}, \mathrm{X} \in \mathrm{G}$,

$$
\rho_{\lambda}(\operatorname{Exp}(\mathrm{tX})(\psi))=\mathrm{C}^{-\lambda} \rho(\operatorname{Exp}(\mathrm{tX}))\left(\mathrm{C}^{\lambda} \psi\right)
$$


Suppose that another parameter $s$ can be introduced as a function of $t$, such that:

(a) As $t \rightarrow \infty, s \rightarrow 1$

(b) There is a real-valued function $a(s, \lambda)$ such that $\rho(\operatorname{Exp}(\mathrm{t}(\mathrm{s}) \mathrm{X}))(\mathrm{C} \lambda \psi) / \mathrm{a}(\mathrm{s}, \lambda)$ is non-singular at $\mathrm{s}=1$.

Then

$$
\rho_{\lambda}(\operatorname{Exp}(t X))(\psi) a(s(t), \lambda)
$$

takes a definite, computable value as $t \rightarrow \infty$, so that this formula determines the asymptotic behavior, in the classical sense, of matrix elements as $t \rightarrow \infty$, with $\lambda$ held fixed.

Now, let us examine the asymptotic behavior of $\lambda \rightarrow \infty$, with $t$ held fixed. We know that :

$$
\rho_{\lambda}(\mathrm{X})=\rho(\mathrm{X})+\lambda \omega(\mathrm{X})
$$

Hence, $\rho_{\lambda}(\mathrm{X} / \lambda)$ is non-singular in the neighborhood of $\lambda=\infty$. Thus, we can expect that

$$
\operatorname{Exp}\left(t \rho_{\lambda}(X)\right)=\rho_{\lambda} \operatorname{Exp}(t X / \lambda)=\rho_{\lambda} \operatorname{Exp}(t / \lambda X)
$$

will behave decently as $\lambda \rightarrow \infty$.

But,

$$
\begin{aligned}
\rho_{\lambda} \operatorname{Exp}\left(\frac{\mathrm{t}}{\lambda} \mathrm{X}\right) & =\mathrm{C}^{-\lambda} \rho \operatorname{Exp}\left(\frac{\mathrm{t}}{\lambda} \mathrm{x}\right) \mathrm{C}^{\lambda} \\
& =\left[\mathrm{C}^{-\lambda} \rho(\operatorname{Exp}(\mathrm{tx})) \mathrm{C}^{\lambda}\right]^{1 / \lambda}
\end{aligned}
$$

We then face the problem of investigating this formula as $\lambda \rightarrow \infty$. Further work on these lines is dependent on doing morc cxplicit calculations in order to obtain a reasonable idea of what to expect in gencral, honce we will defer it to later. 
Another comment about 12.2: If the operators $C^{\lambda}$ and $C^{-\lambda}$ are genuine operators on the Hilbert space and 12.2 held for all $\mathrm{g}$ in $\mathrm{G}, 12.2$ would say that $\rho^{\lambda}$ was equivalent to $\rho$, and, in particular, the value of the Casimir operators of $\mathrm{G}$ would be the same for both representations. For the case of $G=S L(2, R)$, for example, this would be nonsense. There are two remarks to be made about this: First, the operators $\mathrm{C}^{\lambda}$ and/or $\mathrm{C}^{-\lambda}$ are highly singular. (For example, for the case $G=\operatorname{SL}(2, R), H=$ Hilbert space of functions $\psi(\theta), 0 \leq \theta \leq 2 \pi$, $\mathrm{C}^{\lambda}$ is the operator of multiplication by

$$
\left.(\sin \theta)^{\lambda}\right)
$$

Second, the C may not be valid for the whole group G. (For example, for the case $G=S L(2, R)$, it is readily verified that the $C$ changes if $X$ is replaced by Y). The general idea is that the relevant cohomology vanishes when restricted to a suitable subgroup of $G$. This is not surprising: We have seen many examples of this method of "computing" cohomology groups, i.e., choose a suitable subalgebra or subgroup for which the relevant cohomology groups vanish. 


\section{BIBLIOGRAPHY}

1. F. Bruhat, "Sur les représentations induites des groupes de Lie," Bull. Soc. Math. Fr., 84, 97-205 (1956).

2. R. Dashen and M. Gell-Mann, Proceedings of the Third Coral Gables Conference on Symmetry Principles, (W. H. Freeman Company, San Francisco).

3. W. van Est, "Group cohomology and Lie algebra cohomology in Lie groups," Nederl. Akad. Wetensch. Proc. Ser. A. 56, 484-493, 493-504 (1953).

4. L. L. Foldy, "Relativistic particle systems with interaction," Phys. Rev. 122, 275-278 (1961).

5. D. Freedman and J. M. Wang, "Regge poles and unequal mass scattering processes," Phys. Rev. Lett. 17, 569 (1966).

6. H. Furstenberg, "A Poisson formula for semisimple Lie groups," Ann. of Math. 77, 335-386 (1963).

7. I. M. Gelfand and N.J. Vilenkin, Generalized Functions, Vol. 4, (Academic Press, New York, 1964)

8. M. L. Goldberger and C.E. Jones, "Consistency questions raised by simultaneous Mande]s tam and angular momentum analyticity," Phys. Rev. Lett.17, 105 (1966).

9. F. Hadjiannou, "On the use of imaginary mass representation of the Poincare' group in scattering amplitudes," Nuovo Cimento 44a, 185 (1966).

10. S. Helgason, Differential Geometry and Symmetric Spaces (Academic Press, New York, 1962).

11. R. Hermann, Lie Groups for Physicists,(N.A. Benjamin Press, New York, 1966).

12. R. Hermann, "Analytic continuation of group representations, "Comm. Math. Phys. , Part i, 2, 251-270 (1966); Part II, 3, 53-74 (1966); Part III, 3, 75-97 (1966). 
13. E. Inonu and E. P. Wigner, "On the contraction of groups and their representations," Proc. Nat. Acad. Sci. , USA, 39, 510-524 (1953).

14. A. Kurosh, The Theory of Groups, (Chelsea Publishing Company, New York, 1955).

15. E. Nelson, "Analytic vectors," Ann. Math. 70, 572-615 (1959).

16. A. Nijenhuis and R. Richardson, "Deformation of homomorphisms of Lie groups and Lie algebras," to be published, Bull. Am. Math. Soc.

17. E. Roffman, "Complex inhomogeneous Lorentz group and complex angular momentum," Phys. Rev. Lett. $\underline{16}, 210$ (1966). 